N 7324259

SUDAAR NO. 447

STANFORD UNOVIRSOTY

CENVEER FOR SUSTEMS RESESARER

\section{Control Theory for Random Systems}

by

Arthur E. Bryson, Jr.

September 1972

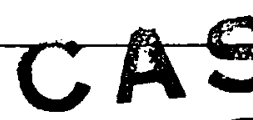

COPY

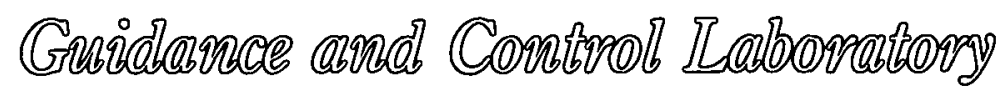

General Lecture for

13th International Congress of

Theoretical and Applied Mechanics

August 21-28, 1972

Moscow, USSR

Prepared under

NAS 2-5143 for the U.S. Army

Air Research and Mobility Command

Ames Directorate

NASA-Ames Research Center

Moffett Field, California 
CONTROL THEORY FOR RANDOM SYSTEMS

\title{
By
}

Arthur E. Bryson, Jr.

\author{
SUDAAR NO. 447 \\ September 1972
}

Guidance and Control Laboratory

General Lecture for

13th International Congress of Theoretical and Applied Mechanics August 21-28, 1972 , Moscow, USSR

Prepared under

NAS 2-5143 for the U. S. Army

Air Research and Mobility Command

Ames Directorate

NASA-Ames Research Center

Moffett Field, California

Department of Aeronautics and Astronautics

Stanford University

Stanford, California 


\section{ABSTRACT}

This paper is a survey of the current knowledge available for designing and predicting the effectiveness of controllers for dynamic systems which can be modeled by ordinary differential equations.

A short discussion of feedback control is followed by a description of deterministic controller design and the concept of system "state." Need for more realistic disturbance models led to use of stochastic process concepts, in particular the Gauss-Markov process. Kolmogorov and Wiener showed how to estimate the state and the mean-square deviation of the state of a stationary stochastic process from noisy measurements. Kalman. and Bucy extended this theory to nonstationary stochastic processes and gave a simpler technique for designing the estimator (or filter) that is well suited for use with digital computers. Feeding back this estimated state with a deterministic "no-memory" controller constitutes a "compensator," a concept familiar in classical controller design. The system, controlled by this compensator, with random forcing functions, random errors in the measurements, and random initial conditions, is itself a Gauss-Markov random process; hence the mean-square behavior of the controlled system is readily predicted. As an example, a compensator is designed for a helicopter to maintain it in hover in a gusty wind over a point on the ground. 
Abstract. ...................................

List of Figures.................................

List of Tables................................

I. INTRODUCTION. ...............................

I I. DESIGN OF STATE-FEEDBACK CONTROLLERS. . . . . . . . . . . . . . 4

III. MODELING RANDOM DISTURBANCES AS STOCHASTIC PROCESSES.... 11

IV. DESIGN OF STATE ESTIMATORS.....................

V. MEAN SQUARE BEHAVIOR OF SYSTEMS WITH FILTER/STATEFEEDBACK CONTROLLERS.......................... 29

VI. $\quad$ SUMMARY .................................

VII. ACKNOWLEDGEMENTS............................... 32

REFERENCES ...................................

BIBLIOGRAPHY ................................ 


\section{LIST OF FIGURES}

1. Block Diagram; Open-Loop (Programmed) Control Has No Feedback Loop, Closed-Loop Control Has Feedback Loop.....

2. Block Diagram; Dynamic Compensator Formed from Filter and state-Feedback Gains......................

3. Nomenclature and Reference Axes for Helicopter Hover Example....................................

4. Root Locus for Position Regulator, Helicopter in Hover, with $A_{x} / B$ as Parameter.......................

5. Pendulum in Random Wind of Spectral Density $Q$; Variance and Covariance Histories, starting with Zero Variance..................................

6. Pendulum in Random Wind; Mean Angular Velocity (q) vs. Angular Deflection $(\theta)$, with $87 \%$ Probability Ellipses at Selected Points on Path....................

7. (a) Stationary Time Correlation; First Order System Forced by Gaussian White Noise, w, with Spectral Density $Q$, (b) Stationary Time Correlations and Cross-Correlation; Second. Order Underdamped System Forced by Gaussian White Noise, w, with Spectral Density $Q$ (e.g., Pendulum in Random Wind)..........

8. Pendulum in Random Wind - Estimate Error Variance and Covariance Histories Using Measurement of $q$ with Additive White Noise Having Spectral Density $\mathrm{R}$.......

9. Root Locus for $(x, \theta)$ Measurement Filter, Helicopter in Hover, with $Q / R_{\theta}$ as Parameter, $\left(R_{x} / R_{\theta}\right)=$ $5.6 \times 10^{-5} \mathrm{~m}^{-2}$ 


\section{LIST OF TABLES}

$\underline{\text { Page }}$

1. Regulator Design for a Typical Transport Helicopter...... 44

2. Filter Design for a Typical Transport Helicopter........ 45

3. Dynamic Compensator for a Typical Transport Helicopter... 46

4. Predicted RMS State and Control Deviations of Typical

Transport Helicopter in a Gusty Wind using Controller

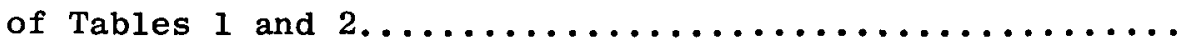




\section{INTRODUCTION}

Automatic control is used in connection with goal-oriented, man-made systems. There are several, usually overlapping, reasons for using automatic control: (a) to relieve human operators from tedious, repetitive tasks (e.g., dial telephones, traffic signals, automatic elevators, autopilots), (b) to speed up and/or lower costs of production processes (e.g., chemical process control, automatic cutting and drilling machines), and (c) to control rapidly-changing or complicated systems accurately and safely (e.g., guidance and control of spacecraft, automatic landing of aircraft).

There are two distinctly different types of automatic control: programmed (or open-loop) control and feedback (or closed-loop) control (see Figure 1). The difference is that the output affects the signals to the actuator when using feedback control but does not affect them when using programmed control. Typical programmed control systems are those used with traffic signals or automatic washing machines where a sequence of events is scheduled by a clock-timer. Examples of simple feedback control systems are the temperature controllers used in buildings, ovens, and hot water heaters, where the temperature error (the difference between sensed and desired temperature) is used with some controller logic, to switch on and off the heating element.

The purpose of feedback control is to reduce to acceptable levels the effects of unpredictable disturbances and uncertain parameters, using acceptable levels of control action. Thus, it is important for the 
designer of a feedback control system to have reasonably accurate models of (a) the system to be controlled and (b) the expected disturbances. Until recently, engineers had better tools for modeling the system than they had for modeling the expected disturbances; they used only rather simple descriptions such as constant values and impulse functions (or integrals of the latter--step functions, ramp functions, etc.). While such descriptions are often adequate to yield satisfactory designs when only one disturbance source acts on the system, more realistic descriptions are needed when several disturbance sources act on the system simultaneous1y.

Many systems of practical interest can be modeled by linear ordinary differential equations. A convenient form for such a model is a set of coupled first-order ordinary equations:

$$
\begin{aligned}
& \dot{x}=F(t) x+G(t) u+\Gamma(t) w ; x\left(t_{0}\right)=x_{0}, \\
& y=M(t) x, \quad \xi=N x\left(t_{f}\right), \\
& z=H(t) x+v,
\end{aligned}
$$

where

$$
\begin{aligned}
x(t) & =n \text {-vector of state variables, } \\
u(t) & =\text {-vector of control variables, } \\
w(t) & =\text {-vector of process disturbance variables, } \\
\xi & =\text {-vector of terminal state parameters }(s \leq n), \\
y(t) & =\text {-vector of output variables }(p \leq n), \\
z(t) & =\text {-vector of measurement variables, } \\
v(t) & =\text {-vector of measurement disturbance variables, } \\
F(t) & =\text { n×n open-loop dynamics matrix, } \\
G(t) & =n \times m \text { control distribution matrix, }
\end{aligned}
$$




$$
\begin{aligned}
\Gamma(t) & =n \times r \text { disturbance distribution matrix, } \\
M(t) & =\text { p } \times n \text { output distribution matrix, } \\
N & =\text { s } \times n \text { terminal state distribution matrix, and } \\
H(t) & =q \times n \text { measurement distribution matrix. }
\end{aligned}
$$

In most problems, the vectors are deviations from nominal values and the matrix elements are partial derivatives evaluated on the nominal "path." The control problem is to design a dynamic system (a "compensator") with measurements, $z$, as input and controls, $u$, as output, so that $y(t)$ and $\xi$ of the controlled system are kept acceptably close to zero in the presence of anticipated disturbances $w, v$, and $x_{0}$, while using acceptable amounts of control, $\mathbf{u}$.

During the last ten years it has become clear that excellent compensators can be designed in two completely separate steps: (a) a statefeedback controller, $u=-C x$, is designed to minimize an appropriate quadratic error criterion, assuming the availability of perfect measurements of the system state variables and no disturbances, i.e., $z=x$, $v=0, w=0 ;(b)$ a measurement-feedback estimator (or filter) is designed to yield maximum likelihood estimates of the state variables of the system, $\hat{x}$, from the available sensor measurements, $z$, taking into account $w, v, x_{0}$, and assuming perfect knowledge of the control variables, $u(t)$. The state-feedback controller is then used with the state estimates from the filter, $i . e ., u=-c(t) \hat{x}$. The resulting control system forms a "dynamic compensator" (see Figure 2) which minimizes the quadratic error criterion on the average [Joseph and Tou (1961), Gunckel and 
Franklin (1963), and Potter (1964)].*

In Section II we discuss state feedback controller design, and in Section IV we discuss the design of state estimators (measurementfeedback filters). Section III is a review of the concept of a GaussMarkov random process which forms the basis of the filter design. Section $V$ discusses the behavior of systems controlled by such filter/statefeedback compensators.

\section{DESIGN OF STATE-FEEDBACK CONTROLLERS}

For $w=v=0$, and $z \equiv x$, a rather general design problem for the system (1.1)-(1.2) is to try to find an acceptable $u(t)$ program in some time interval, $t_{0} \leq t \leq t_{f}$, so that the output variables, $y(t)$ and $\xi$ stay acceptably close to zero for a given $x_{0}$.

A useful way to judge one design relative to another is to specify a quadratic criterion of the form:

$$
J=\frac{1}{2} \xi \Omega_{\partial \xi}+\frac{1}{2} \int_{t_{0}}^{t_{f}}\left(y^{T} A y+u^{T} B u\right) d t
$$

where the positive definite weighting matrices $\Omega, A(t)$, and $B(t)$ are chosen to express relative preferences of the designer, and []$^{T}$ indicates matrix transpose of [ ], i.e., interchange rows and columns. Using the calculus of variations (or dynamic programming), it is straightforward to show that the state-feedback controller that minimizes

\footnotetext{
* Joseph and Tou, as well as Gunckel and Franklin, showed this for GaussMarkov sequences (discrete-step processes); Potter extended the proof to continuous Gauss-Markov processes.
} 
$\mathrm{J}$ is given by:*

$$
u=-c(t) x
$$

where the $m \times n$ feedback gain matrix, $c(t)$, is given by

$$
C=B^{-1} G^{T} S
$$

and

$$
\dot{S}=-S F-F^{T} S+C^{T} B C-M^{T} A M ; S\left(t_{f}\right)=N^{T} \partial 2 N
$$

The nonlinear equation, (2.4), for the symmetric $m \times n$ matrix, $s(t)$, is called a backward matrix Ricatti equation because of the term $c^{\mathrm{T}} \mathrm{BC}=$ $S G B^{-1} G^{T} S$ which is quadratic in the elements of $S$, and because it must be integrated backward from the final time, $t_{f}$, to the initial time, $t_{0}$, to generate $c(t)$. The significance of $s$ is that

$$
J_{\min }=\frac{1}{2} x^{T}\left(t_{0}\right) S\left(t_{0}\right) x\left(t_{0}\right)
$$

Examples of such time-varying terminal control problems and their solutions are given elsewhere [e.g., Bryson and Ho (1969), Section 14.6].

Obviously it is not always possible to control all of the outputs with a given set of controls. A "controllability" criterion was given by Kalman, Ho, and Narendra (1963).

Of special interest is the class of problems with stationary solutions. For such problems, the matrices F, G,M,A,B must be constant, and $t_{f^{-}} t$ must be large compared to characteristic times of the controlled system. S may then have a constant asymptotic value, which means that the feedback gain matrix tends to a constant value:

* See, e.g., Bryson and Ho (1969), Section 5.2 If $x\left(t_{0}\right)$ is known ahead of time, the optimum $u(t)$ can be pre-calculated and programmed as an open-loop input; this is a peculiar feature of deterministic control design--it predicts no difference between open-1oop and closed-loop control. 


$$
\left.\begin{array}{l}
S(t) \rightarrow S_{\infty} \\
C_{\infty}=B^{-1} G^{T} S_{\infty}
\end{array}\right\} \text { as } t_{f}-t \rightarrow \infty
$$

A controller of the form (2.2) with constant gains, $C_{\infty}$, is called a regulator. In principle, the regulator gains $C_{\infty}$ could be found by solving sets of quadratic equations [Eqs. (2.4) with $\dot{\mathrm{S}}=0$ ] to find the elements of the steady-state matrix, $s_{\infty}$. However, this turns out to be an impractical procedure for $n \geq 3$. Until recently, the procedure most often used was to integrate the matrix Ricatti equation, (2.4), backward on a computer until $S(t)$ reached a steady-state [see, e.g., Kalman and Englar (1966)]. This turns out to be expensive and involves several numerical difficulties and hazards. MacFarlane (1963) and Potter (1966) showed another ingenious way to find $S_{\infty}$ using eigenvector decomposition. It does not seem to be widely known or used, perhaps because it requires an accurate computer program to find the eigenvalues and eigenvectors of a large $(2 n \times 2 n)$ matrix. However, Francis (1962) developed a remarkably fast and accurate algorithm for eigenvalue-eigenvector determination (the "QR algorithm"), and Hall (1971) applied it to the MacFarlane-Potter method for finding $s_{\infty}$.

The eigenvector decomposition method is based on finding the eigenvectors of the Euler-Lagrange equations for the problem of minimizing (2.1):

$$
\left[\begin{array}{l}
\dot{x} \\
\dot{\lambda}
\end{array}\right]=\left[\begin{array}{c}
\mathbf{F},-G^{-1} G^{T} \\
-M_{A M}^{T},-F^{T}
\end{array}\right]\left[\begin{array}{l}
\mathbf{x} \\
\lambda
\end{array}\right]
$$

The eigenvalues of this system are symmetric about the imaginary axis in the complex plane. (If $s_{i}$ is an eigenvalue, $-s_{i}$ is also an eigenvalue.) 
Hence, the characteristic equation of $(2.8)$ is called the symmetric-root characteristic equation [see Letov (1960), Chang (1961), and Rynaski and Whitbeck (1966)]. If we let $T$, be the $2 n \times n$ matrix consisting of the n eigenvectors of $(2.8)$ corresponding to the eigenvalues with negative real parts, then Potter (1966) has shown that:

$$
S_{\infty}=\Lambda_{-}\left(x_{-}\right)^{-1} \text { where } T_{-}=\left[\begin{array}{l}
x_{-} \\
\Lambda_{-}
\end{array}\right] \text {. }
$$

Furthermore, the eigenvalues of the controlled system (i.e., the eigenvalues of $F-G C$ ) are the eigenvalues of (2.8) that have negative real parts, and the eigenvectors of the controlled system are the columns of $x_{-}$.

A revealing alternative form for (2.8), in terms of the Laplace transform of the control variables, $u(s)$, was suggested by Rynaski and Whitbeck (1966):

$$
\left[\mathrm{B}+\mathrm{Y}^{\mathrm{T}}(-\mathrm{s}) \mathrm{AY}(\mathrm{s})\right] \mathrm{u}(\mathrm{s})=0
$$

where $Y(s)=M[s I-F]^{-1} G$ is the matrix of transfer functions from the control variables to the outputs, i.e.,

$$
y(s)=Y(s) u(s)
$$

and. $s$ is the Laplace transform variable. The corresponding equation for the system using feedback control gains, C, may be written

$$
\left|I+C(s I-F)^{-1} G\right| u(s)=0 \text {. }
$$

It follows from $(2.10)$ and (2.12) that

$$
\left[B+Y^{T}(-s) A Y(s)\right]=\left|I+C(-s I-F)^{-1} G\right|^{T} B\left[I+C(s I-F)^{-1} G\right]
$$

which is an mXm matrix equation (where $u$ is an m-vector). For $s=i \omega$, 
where $\omega=$ frequency, $(2.13)$ is the well-known "spectral factorization" problem encountered in solving certain Wiener-Hopf integral equations. The problem here is to find the mxn matrix $C$ that satisfies (2.13), i.e., to "factor" the left side of (2.13) into two m $\times n$ matrices, one with eigenvalues in the left half of the complex $s$ plane and the other with exactly the negative of those eigenvalues which are then in the right half plane.

This factorization can be done very quickly and accurately by eigenvector decomposition, using (2.8) and a digital computer [see, e.g., Bryson and Hall (1971)]. It can also be done as follows: (a) find the $n$ eigenvalues of $(2.8)^{*}$ that have negative real parts and the corresponding $n$ eigenvectors of (2.10) (each with only $m$ components); then (b), require that the eigenvalues and eigenvectors of $(2.12)$ be the same as those in (a). If $m=1$, all that is needed is to equate coefficients of like powers of $s$ of the characteristic equation of (2.12) and the closed-1oop characteristic equation formed from the $n$ eigenvalues of (2.8) that have negative real parts; this gives $n$ linear equations for the $n$ components of $c$. If $m>1$, the requirement that the eigenvectors be parallel adds $n(m-1)$ equations, giving a total of $n m$ linear equations for determining the $\mathrm{nm}$ components of $\mathrm{C}$.

As an example, we design a regulator for a fourth-order model of a helicopter in hover. Figure 3 shows the nomenclature and reference axes. The four state variables are $\theta=$ pitch angle of the fuselage, $q=$ pitch

\footnotetext{
*For $\mathrm{m}>1$, the characteristic equation of $(2.10)$ is of order $2 \mathrm{mn}$ instead of $2 \mathrm{n}$, and hence contains extraneous roots (pointed out to author by J. V. Breakwe11).
} 
rate $(q=\dot{\theta}), \mathbf{x}=$ horizontal distance of mass center from the hover point, and $u=$ horizontal velocity $(u=\dot{x})$. The control variable is $\delta=$ tilt angle of the rotor-thrust vector with respect to the fuselage. The primary disturbance is horizontal wind, w, which we shall consider in a later section. The vertical motions (controlled by increasing or decreasing rotor thrust), the lateral motions (controlled by tilting the rotor thrust laterally), and the yawing motions (controlled by the tail rotor) are all nearly uncoupled from the longitudinal motions considered here and may be treated as separate regulator design problems. The longitudinal equations of motion in state variable form may be written as:

$$
\left[\begin{array}{c}
\dot{\theta} \\
\dot{q} \\
\dot{u} \\
\dot{x}
\end{array}\right]=\left[\begin{array}{cccc}
0 & 1 & 0 & 0 \\
0 & -\sigma_{1} & -a_{1} & 0 \\
g & -a_{2} & -\sigma_{2} & 0 \\
0 & 0 & 1 & 0
\end{array}\right]\left[\begin{array}{l}
\theta \\
q \\
u \\
x
\end{array}\right]+\left[\begin{array}{l}
0 \\
n \\
g \\
0
\end{array}\right] \delta+\left[\begin{array}{c}
0 \\
-a_{1} \\
-\sigma_{2} \\
0
\end{array}\right] w
$$

A reasonable quadratic performance index is:

$$
J=\frac{1}{2} \int_{0}^{\infty}\left(A_{x} x^{2}+B \delta^{2}\right) d t
$$

which reflects a desire to keep $x$ and $\delta$ near zero.

The root square characteristic equation in the form $(2.10)$ is given by:

$$
B+A_{X} Y_{X}(s) Y_{X}(-s)=0
$$

where

$$
\begin{gathered}
Y_{x}(s)=\frac{x(s)}{\delta(s)}=\frac{g\left[s^{2}+\left(\sigma_{1}-\frac{a_{2} n}{g}\right) s+n\right]}{\Delta_{0}(s)} \\
\left.\Delta_{0}(s)=s \mid s^{3}+\left(\sigma_{1}+\sigma_{2}\right) s^{2}+\left(\sigma_{1} \sigma_{2}-a_{1} a_{2}\right) s+a_{1} g\right]
\end{gathered}
$$


and $\Delta_{0}(s)=0$ is the open-loop characteristic equation. Thus $(2.16)$ becomes:

$\Delta_{0}(s) \Delta_{0}(-s)+\frac{A_{x}}{B} g^{2}\left[s^{2}+\left(\sigma_{1}-\frac{a_{2} n}{g}\right) s+n\right]\left[s^{2}-\left(\sigma_{1}-\frac{a_{2}{ }^{n}}{g}\right)+n\right]=0$.

Coefficients for a typical transport helicopter are given in Table 1 , along with the open-loop eigenvalues and eigenvectors. Note the uncontrolled helicopter is slightly unstable in hover, which is fairly typical. The weighting factor $A_{x / B}$ may be selected as follows: rewrite (2.15) as

$$
J=\frac{1}{2} \int_{\delta}^{\infty}\left[\left(\frac{x}{x_{0}}\right)^{2}+\left(\frac{\delta}{\delta_{0}}\right)^{2}\right] d t
$$

Suppose $\delta_{0}=5^{\circ}$ is the maximum amount of rotor tilt we would like to commit to position control, and we would be willing to use this amount when the position error is 2 meters. It follows then that we should choose $\ddot{0}=5.0 / 57.3 \mathrm{rad}$ and $x_{0}=2.0$ meters. Hence

$$
\frac{A_{x}}{B}=\frac{\delta_{0}^{2}}{x_{0}^{2}}=\left(\frac{5.0}{57.3}\right)^{2} \frac{1}{(2)^{2}} \cong .0020 \mathrm{~m}^{-2}
$$

It is often helpful to the designer to see how the eigenvalues change as the weighting factors change. The concept of a root locus plot was introduced by Evans (1950); Chang (1961) applied it to the root square characteristic equation (2.10) and Rynaski and Whitbeck (1965) extended the application to multi-input, multi-output systems. Figure 4 shows a root locus of $(2,17)$ with $A_{x / B}$ as the parameter. The open-loop poles and their reflections across the imaginary axis are the "poles" (shown as $x$ 's ) of the root locus; the "zeros" (shown as 0 's ) are at $\pm\left(.25 \pm 2.50 j\right.$ ) $\mathrm{sec}^{-1}$. 
- For the weighting factor given in (2.18) the closed-loop eigenvalues and eigenvectors, as well as the corresponding feedback gains, are given in Table 1 .

Even for $A_{x}=0,(B \neq 0)$ the $\theta, q, u$ states of the helicopter are stabilized by this technique; the two unstable complex roots of the open-loop system are simply "reflected" across the imaginary axis, while the stable real root and the zero root remain unchanged. The corresponding gains are:

$$
c_{\theta}=.0535, c_{q}=.0785 \text { sec. }, c_{u}=c_{x}=0 \text {. }
$$

\section{MODELING RANDOM DISTURBANCES AS STOCHASTIC PROCESSES}

How can "unpredictable disturbances" be modeled realistically? one very useful way is to describe them as stochastic processes, a concept which was largely developed in this century by Einstein ("Brownian motion," 1905), Langevin (1908), Markov (1912), Planck (1917), Wiener (1930), Uhlenbeck and Ornstein (1930), Kolmogorov (1931), Khintchine (1938), Wold (1938), Chandrasekar (1943), Rice (1944), Wang and Uhlenbeck (1945), Phillips (1947), Laning and Battin (1958), Newton, Gould, and Kaiser (1957), and Kalman and Bucy (1961), to mention only a few.

A special class of stochastic processes, the Gauss-Markov process (GMP), has emerged as a particularly simple and useful one in describing random disturbances. Many natural and man-made dynamic processes can be modeled quite well by GMP's. A GMP may be described by giving its mean value vector, $\bar{x}(t)$, and its correlation matrix $c(t, \tau)$, where 


$$
\begin{aligned}
\bar{x}(t) & \triangleq E[x(t)] \\
C(t, \tau) & \triangleq E\left\{[x(t)-\bar{x}(t)][x(\tau)-\bar{x}(\tau)]^{T}\right\},
\end{aligned}
$$

E[ ] means "expected value of," and $x$ is a column vector with $n$ components. The operation on the right side of (3.2) is an "outer product" or "dyadic product"; in Cartesian tensor notation.

$$
\left.\left.C_{i j}(t, \tau) \triangleq E\left\{\mid x_{i}(t)-\bar{x}_{i}(t)\right\} \mid x_{j}(\tau)-\bar{x}_{j}(\tau)\right]\right\}
$$

The "expected value of [ ] " may be thought of as an "ensemble average," i.e., the average of [ ] over a large number of samples of the random process $x(t)$. If $x^{(i)}(t)$ is the $i^{\text {th }}$ sample history, then

$$
\begin{aligned}
\bar{x}(t) & =\lim _{N \rightarrow \infty} \frac{1}{N} \sum_{i=1}^{N} x^{(i)}(t) \\
c(t, \tau) & =\lim _{N \rightarrow \infty} \frac{1}{N} \sum_{i=1}^{N}\left[x^{(i)}(t)-\bar{x}(t)\right]\left[x^{(i)}(\tau)-\bar{x}(\tau)\right]^{T}
\end{aligned}
$$

The covariance matrix, a measure, of the mean-square de viat io $n$ of the random process from its mean value, is given by

$$
x(t) \triangleq E\left\{[x(t)-\bar{x}(t)][x(t)-\bar{x}(t)]^{T}\right\}
$$

Obviously $x(t)=c(t, t)$. The probability density of $x(t)$ at any time $t$ is gaussian; i.e.,

$$
p[x(t)]=\frac{1}{(\pi)^{\frac{n}{2}}|x(t)|^{\frac{1}{2}}} \exp \left\{-\frac{1}{2}[x(t)-\bar{x}(t)]^{T} x^{-1}(t)[x(t)-\bar{x}(t)]\right\}
$$

where $p(\xi) d(\xi)$ is the probability that the value of $x$ lies between $\xi$ and $\xi+d \xi$. 
The basic GMP is the purely-random process; it is an idealized, very jittery process that is completely uncorrelated from one time to the next. Its correlation matrix has the form

$$
Q(t) \delta(t-\tau)
$$

where $\delta(\cdot)$ is the Dirac delta function. The purely random process may be thought of as the limit of a sequence of impulses, random in both magnitude and time of occurrence, where the average magnitude of the impulses is zero (as many positive as negative impulses), but the mean square magnitude is $[\sigma(t)]^{2}$. The power spectral density is then

$$
Q(t) \stackrel{\sim}{=}[\sigma(t)]^{2} \beta(t)
$$

where $\beta(t)$ is the average number of impulses per unit time that occur at time $t$.

A GMP can always be represented by the state vector, $x(t)$, of a linear dynamic system forced by a gaussian purely random process where the initial state vector is gaussian:

$$
\dot{x}=F(t) x+\Gamma(t) w
$$

where

$$
\begin{aligned}
& E[w(t)]=\bar{w}, E\left\{\left[w(t)-\bar{w}^{\prime}\left[w(\tau)-\bar{w}^{T}\right\}=Q(t) \delta(t-T)\right.\right. \\
& E\left[x\left(t_{0}\right)\right]=\bar{x}_{0}, E\left\{\left[x\left(t_{0}\right)-\bar{x}_{0}\right]\left[x\left(t_{0}-\bar{x}_{0}\right]^{T}\right\}=x_{0},\right. \\
& E\left\{[w(t)-\bar{w}]\left[x\left(t_{0}\right)-\bar{x}_{0}\right]\right\}=0 .
\end{aligned}
$$

This markov representation shows clearly that the state property for 
deterministic processes and the markov property for random processes are precisely analogous: (a) if knowledge of a finite-dimensional vector at any time, $t_{0}$, makes the future of a deterministic process independent of the past, then it has the state property. For example, if $w(t)$ in $(3.8)$ is known, then knowledge of $x\left(t_{0}\right)$, the initial state vector, is sufficient to predict $x(t)$ for $t>t_{0}$; (b) if knowledge of the probability density function for a finite-dimensional vector at any time, $t_{0}$, makes the future of a random process independent of the past, then it has the markov property. For example, if $Q(t)$ in (3.8) is known, then knowledge of $\mathrm{p}\left[\mathrm{x}\left(\mathrm{t}_{\mathrm{o}}\right)\right]$, the initial probability density function, is sufficient to predict $p[x(t)]$ for $t>t_{0}$.

Since the gaussian probability density of a vector, $x$, is completely specified by giving its mean value $\bar{x}$ and its covariance matrix, $X$ (see 3.6), a GMP is specified for $t \geq t_{0}$ by giving $\bar{x}\left(t_{0}\right), x\left(t_{0}\right), F(t), \Gamma(t), \bar{w}(t)$, and $Q(t)$. In fact, it can be shown that

$$
\begin{aligned}
& \dot{\bar{x}}=\overline{F \bar{x}}+\Gamma \overline{\mathrm{w}} ; \overline{\mathrm{x}}\left(\mathrm{t}_{0}\right)=\overline{\mathrm{x}}_{0}, \\
& \dot{\mathrm{x}}=\mathrm{FX}+\mathrm{XF}^{\mathrm{T}}+\Gamma \mathrm{Q} \Gamma^{\mathrm{T}}, \mathrm{x}\left(\mathrm{t}_{\mathrm{o}}\right)=\mathrm{x}_{0} \cdot^{*}
\end{aligned}
$$

Note that $\bar{x}(t)$ and $x(t)$ are completely independent of each other. It can also be shown (e.g., Bryson and Ho, p. 333) that the correlation matrix is given by:

$$
\mathrm{C}(\mathrm{t}, \tau)=\left\{\begin{array}{l}
\Phi(t, \tau) \mathrm{x}(\tau) ; \mathrm{t} \geq \tau, \\
\mathrm{x}(\mathrm{t}) \Phi^{\mathrm{T}}(\tau, \mathrm{t}) ; \mathrm{t} \leq \tau,
\end{array}\right.
$$

\footnotetext{
* If $F=$ constant matrix with eigenvalues $s_{i}, i=1, \ldots, n$, then it can be shown that the $\frac{1}{2}(n+1)$ eigenvalues of $(3.10)$ are all possible combinations $s_{i}+s_{j}, i, j=1, \ldots, n$.
} 
where $\omega(t, \tau)$ is the $n \times n$ transition matrix of $\dot{x}=F x, i . e .$,

$$
\frac{d}{d t} \Phi(t, \tau)=F(t) \Phi(t, \tau) ; \Phi(\tau, \tau)=I
$$

One of the simplest nonstationary GMP's is the so-called Wiener process, which is the integral of stationary white noise:

$$
\begin{aligned}
\dot{x} & =w \\
E[x(0)] & =0, E[x(0)]^{2}=0, \\
D[w(t)] & =0, E[w(t) w(\tau)]=Q \delta(t-\tau), Q=\text { constant. }
\end{aligned}
$$

From (3.9) and (3.10)

$$
\begin{aligned}
& \dot{\bar{x}}=0, \bar{x}(0)=0 \\
&(t)=Q, x(0)=0
\end{aligned} \Rightarrow\left\{\begin{array}{l}
\bar{x}(t)=0 \\
x(t)=Q t .
\end{array}\right.
$$

The root-mean-square (RMS) value $x(t)$ is $\sqrt{x(t)}=\sqrt{Q t}$, which shows the diffusive or random walk character of GMP's. The Wiener process may be thought of as a series of random step functions of average magnitude zero occurring at random times but with a certain average number of steps per unit time, $\beta$. It follows that $Q=2 c^{2} \beta$ where $c^{2}$ is the mean square magnitude of the step functions.

Another well-known nonstationary GMP is the underdamped oscillator forced by white noise [Wang and Uhlenbeck (1945)]. This process could be interpreted, for example, as a pendulum in a random wind (see sketch below). The equations of motion in state variable form may be written as

$$
\begin{aligned}
& \dot{\theta}=q \\
& \dot{q}=-\omega^{2} \theta-2 \xi \omega q+w
\end{aligned}
$$

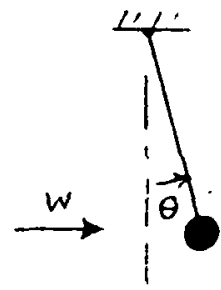


where $w(t)$ is proportional to wind velocity, $\omega=$ undamped natural frequency of the pendulum, and $\zeta=$ damping ratio. Suppose the motion starts with $\theta(0)=\theta_{0}, q(0)=0$ and $\bar{w}(t)=0, E[w(t) w(\tau)]=$ $Q \delta(t-\tau)$, i.e., we know the initial state exactly $\left[x_{\theta \theta}(0)=x_{q q}(0)=x_{q \theta}(0)=0\right]$ and the wind has zero mean velocity but has a constant spectral density proportional to $Q$. The mean value history is given by the familiar equations:

$$
\begin{aligned}
\dot{\theta} & =\bar{q} ; \bar{\theta}(0)=\theta_{0}, \\
\dot{\bar{q}}=\omega^{2} \theta-2 \zeta \omega \overline{\mathrm{q}} ; \overline{\mathrm{q}}(0) & =0 .
\end{aligned}
$$

The elements of the covariance matrix are given by

$$
\begin{aligned}
& \dot{x}_{\theta \theta}=2 x_{\theta q} ; x_{\theta \theta}(0)=0, \\
& \dot{x}_{\theta q}=-\omega^{2} x_{\theta \theta}-2 \xi \omega x_{\theta q}+x_{q q} ; x_{\theta q}(0)=0, \\
& \dot{x}_{q q}=-2 \omega^{2} x_{\theta q}-4 \xi \omega x_{q q}+Q ; x_{q q}(0)=0 .
\end{aligned}
$$

For $\zeta=0.2$, the time histories are shown in Figure 5. Note the two variances and the covariance change with twice the frequency that $\bar{q}$ and $\bar{\theta}$ change. A more revealing display of the time histories is given in Figure 6 , where $\bar{q} / \omega$ is plotted against $\bar{\theta}$ and probability ellipses are shown at selected points on the path. The axes of the ellipses are in the directions of the eigenvectors of $x$ and the major and minor axes are proportional to the eigenvalues of $x$. Note the angular velocity $q$ becomes uncertain first (elongated ellipse parallel to $q$ axis) but the uncertainty quickly extends to $\theta$. For $\sqrt{1-\zeta^{2}} \omega t \gg 2 \pi$, 


$$
\bar{\theta} \rightarrow 0, \bar{q} \rightarrow 0, x_{\theta q} \rightarrow 0, x_{\theta \theta} \rightarrow \frac{Q}{4 \xi \omega}{ }^{3}, x_{q q} \rightarrow \frac{Q}{4 \xi \omega} .
$$

Thus, it is the presence of damping $(\zeta>0)$ that produces a statistical steady-state. The uncertainty created by the random wind is just balanced by the effects of damping. Note that $x$ does not always increase with time; if $x(0)>x(\infty)$, then $x$ would decrease with time toward $x(\infty)$.

\section{Stationary Gauss-Markov Processes}

For a stationary GMP,

$$
c(t, \tau)=C(t-\tau),
$$

that is, $C$ is a function of $(t-\tau)$ only. The Fourier transform of the correlation matrix of a stationary GMP is called its spectral density matrix, $Q(\omega)$, i.e. ,

$$
Q(\omega) \triangleq \int_{-\infty}^{\infty} c(t) e^{-j \omega t} d t
$$

which implies that

$$
C(t)=\frac{1}{2 \pi} \int_{-\infty}^{\infty} Q(\omega) e^{j \omega t} d \omega
$$

The scalar, stationary, purely random GMP has a constant spectral density and for this reason is often called white noise, since it is made up of all frequencies from 0 to ${ }^{\infty}$. Its correlation is therefore

$$
Q 6(t-T)
$$

where $Q=$ spectral density (constant). 
At the opposite extreme from white noise is a random bias; it is simply an unpredictable constant. It has a constant correlation and hence its spectral density is given by

$\mathrm{C} \delta(\omega)$

where. $\mathrm{C} / 2 \pi=$ correlation (constant). Its spectrum is thus a "line" at $w=0$ (zero frequency). In between white noise (zero correlation time), and a random bias (infinite correlation time), stationary GMP's have finite correlation times, i.e., $\mathrm{C}(\mathrm{t}-\tau) \rightarrow 0$ as $|\mathrm{t}-\tau| \rightarrow \infty$. For example, white noise forcing a first-order system produces a GMP with an exponential correlation (see Figure 7). As another example, the components of the correlation matrix for the pendulum in a random wind (when it has reached a statistical steady state) are also shown in Figure 7 for $\zeta=0.2$.

\section{DESIGN OF STATE EST IMATORS}

In the early $1960^{\prime}$ s a significant advance was made in control theory for random systems. Kalman and Bucy (1961), using the calculus of variations, developed the maximum likelihood filter for estimating the state of a nonstationary GMP in real time from measurements of the process that contain additive white noise. This extended and clarified the pioneering work of Wiener (1942) and Kolmogorov (1941), who had developed the minimum meansquare error filter for stationary stochastic processes (not necessarily markovian or gaussian) using generalized harmonic analysis.

Kalman and Bucy assumed that a mathematical model of the GMP to be estimated was known, (1.1), and assumed that the process noise, w(t), and the measurement noise, $v(t)$, were purely random processes:

\footnotetext{
* Note that if $\mathrm{q}<\mathrm{n}, \mathrm{z}(\mathrm{t})$ is, in general, not a markov process since $z\left(t_{0}\right)$ does not enable one to predict $z(t) \overline{\text { for }} t>t_{0}$. 


$$
\begin{aligned}
& E[w(t)]=0, E\{[w(t)][w(\tau)]\}^{\Gamma}=Q(t) \delta(t-\tau) \\
& E[v(t)]=0, E\{[v(t)][v(\tau)]\}^{\Gamma}=R(t) \delta(t-\tau)^{*}
\end{aligned}
$$

where $Q(t)$ is a known, possibly time-varying, $r \times r$ spectral density matrix for the process noise and $R(t)$ is a known, possibly time-varying, $q \times q$ spectral density matrix for the measurement noise.

The Kalman-Bucy (KB) filter is then given by:

$$
\dot{\hat{x}}=F \hat{x}+G u+K(z-H \hat{x}) ; \hat{x}\left(t_{0}\right)=\bar{x}\left(t_{0}\right)
$$

where

$$
\left.\begin{array}{l}
\mathrm{K}=\mathrm{PH}^{\mathrm{T}} \mathrm{R}^{-1} \\
\dot{\mathrm{P}}=\mathrm{FP}+\mathrm{PF}+\Gamma \mathrm{T} \Gamma^{\mathrm{T}}-\mathrm{KRK}^{\mathrm{T}} ; \mathrm{P}\left(\mathrm{t}_{\mathrm{o}}\right)=\mathrm{x}\left(\mathrm{t}_{\mathrm{o}}\right)
\end{array}\right\} .
$$

Note $F, \Gamma, K, H, P, R, Q$ can all be time-varying quantities, and the process can start at any time, $t_{0} \cdot G u$ is assumed to be a known forcing function. $^{* *}$ The filter (4.2) is similar to the mean value model of (3.9), with the addition of some known forcing functions, $\mathrm{Gu}$, and some feedback terms proportional to $(z-H \hat{x})$, the difference between the actual measurements and the expected value of the measurements based on the current estimate of the state. (The expected value of the measurement noise, $v$, is zero since a purely random process cannot be predicted.) The rate of change of the estimated state, $\dot{\hat{x}}$, is thus proportional to the measurement prediction error.

\footnotetext{
* Obviously, there is no lack of generality in assuming $\bar{w}(t)=\bar{v}(t)=0$. ** If there is uncertainty in implementing $u(t)$, it can be included in $w(t)$ 
The errors in the estimates given by (4.2) are defined as

$$
\tilde{x} \triangleq \hat{x}-\mathbf{x}
$$

By subtracting (1.1) from (4.2), a set of differential equations for the errors in the estimates can be found:

$$
\dot{\tilde{x}}=(F-K H) \tilde{x}+K v-\Gamma w ; \tilde{x}\left(t_{0}\right)=\bar{x}\left(t_{0}\right)-x\left(t_{0}\right) .
$$

From (4.5) it is clear that errors in the estimates are caused by (a) random errors in the measurement signals, $v(t)$; (b) random forcing functions, $w(t)$, and random initial conditions, $x\left(t_{0}\right)$.

The $n \times n$ symmetric matrix $P(t)$ in $(4.3)$ is the covariance matrix of the error in the estimates, i.e.,

$$
P(t)=E\left\{[\hat{x}(t)-x(t)][\hat{x}(t)-x(t)]^{T}\right\} \text {. }
$$

It may be computed from (4.3), which is a forward matrix Riccati equation, since $\mathrm{KRK}^{\mathrm{T}} \equiv \mathrm{PH}^{\mathrm{T}} \mathrm{R}^{-1} \mathrm{HP}$ is quadratic in the elements of $\mathrm{P}$. Comparing (4.3) with (3.10) we see that they are the same except for the information rate term $\left(-\mathrm{KRK}^{\mathrm{T}}\right)$. Obviously $\mathrm{P}$ is less than or equal to. $\mathrm{X}$, i.e., with measurement information the uncertainty in the state is usually reduced.

The $n \times q$ gain matrix, $K(t)$, is proportional to the uncertainty in the estimate of the state (the covariance matrix, $P$ ) and inversely proportional to the uncertainty in the measurements (the spectral density matrix $R$ ) . Thus, if the uncertainty in state is large compared to uncertainty in measurements, the filter weights the measurement prediction error heavily and vice-versa. 
Obviously it is not always pussible to estimate all the state variables from a given set of measurements (4.1). An "observability" criterion was given by Kalman, Ho, and Narendra (1963).

As an example, suppose we had a measurement of the angular velocity of the pendulum in a random wind, i.e.,

$$
\mathbf{z}=\mathbf{q}+\mathbf{v}
$$

so that $H=[0,1]$, and assume $R=$ constant. The $K B$ filter is then

$$
\begin{aligned}
& \dot{\hat{\theta}}=\hat{\mathrm{q}}+\mathrm{K}_{\theta}(\mathrm{z}-\hat{\mathrm{q}}) \quad ; \hat{\theta}(0)=\theta_{0}, \\
& \dot{\hat{\mathrm{q}}}=-\omega^{2} \hat{\theta}-2 \zeta \omega \hat{\mathrm{q}}+\mathrm{K}_{\mathrm{q}}(\mathrm{z}-\hat{\mathrm{q}}) ; \hat{\mathrm{q}}(0)=0,
\end{aligned}
$$

where

$$
K_{\theta}=\frac{1}{R} P_{\theta q}, \quad K_{q}=\frac{1}{R} P_{q q},
$$

and

$$
\begin{aligned}
& \dot{\mathrm{p}}_{\theta \theta}=2 \mathrm{P}_{\theta \mathrm{q}}-\frac{1}{\mathrm{R}} \mathrm{p}_{\theta \mathrm{q}}^{2} \\
& ; \quad P_{\theta \theta}(0)=0 \\
& \dot{\mathrm{P}}_{\theta \mathbf{q}}=-\omega^{2} \mathrm{P}_{\theta \theta}-2 \zeta \omega \mathrm{P}_{\theta \mathbf{q}}+\mathrm{P}_{\mathrm{qq}}-\frac{1}{\mathrm{R}} \mathrm{P}_{\theta \mathbf{q}} \mathrm{P}_{\mathrm{qq}} ; \mathrm{P}_{\theta \mathbf{q}}(0)=0 \\
& \left.\dot{\mathrm{P}}_{\mathrm{qq}}=-2_{\omega}^{2} \mathrm{P}_{\theta \mathrm{q}}-4 \xi \omega \mathrm{P}_{\mathrm{qq}}+\mathrm{Q}-\frac{1}{\mathrm{R}} \mathrm{P}_{\mathrm{qq}}^{2} \quad ; \mathrm{p}_{\mathrm{qq}}(0)=0 .\right)
\end{aligned}
$$

The estimates $\hat{\theta}$ and $\hat{q}$ from (4.8) depend on the measurement history, $z(t)$, of the particular run. However, we can predict $P(t)$, the meansquare error of the estimates, ahead of time. For $\zeta=0.2$ and $Q / \omega^{2} R=$ 2.0 , the time histories of $P_{\theta \theta}, P_{\theta q}, P_{q q}$ are shown in Figure 8 . They are similar to the time histories of $x_{\theta \theta}, x_{\theta q}, x_{q q}$ in Figure 5 but are less. The uncertainty created by the random wind is balanced not only by 
damping but also by the information coming from the measurement of $q$. Again a statistical steady-state is reached for $\sqrt{1-\zeta^{2}} \omega t \gg 2 \pi$ :

$$
\left.\begin{array}{l}
\mathrm{P}_{\theta \mathrm{q}} \rightarrow 0 \\
\mathrm{P}_{\theta \theta} \rightarrow \frac{\mathrm{Q}}{4 \omega^{3}} \frac{2}{\zeta+\zeta^{\prime}} \\
\mathrm{P}_{\mathrm{qq}} \rightarrow \frac{\mathrm{Q}}{4 \omega} \frac{2}{\zeta+\zeta^{\prime}}
\end{array}\right\} \text { where } \zeta^{\prime}=\sqrt{\zeta^{2}+\frac{\mathrm{Q}}{4 \omega^{2} \mathrm{R}}} .
$$

For $Q / \omega^{2} \mathrm{R} \rightarrow 0$ (noise in the measurement $\gg$ wind noise), Equations (4.11) indicate no change from the unmeasured situation (3.18). For $Q / \omega^{2} R \rightarrow \infty$ (noise in the measurement $\ll$ wind noise), $\mathrm{P}_{\theta \theta} \rightarrow 0, \mathrm{P}_{\mathrm{qq}} \rightarrow 0$, i.e., the errors in the estimates become negligibly small.

\section{Stationary Filters}

The Wiener-Kolmogorov (WK) filtering technique for stationary gaussian processes, ${ }^{*}$ while remarkably creative, was difficult to understand for most engineers. It involved solving an integral equation (of the WienerHopf type) for the Fourier transform of a kernel function, $w(t-\tau)$, such that a scalar "signal" is estimated with minimum mean-square error, by

$$
\int_{-\infty}^{t} w(t-\tau) z(\tau) d t
$$

where $z(t)$ is the signal plus noise; the time correlations (hence spectral densities) of the signal and the noise are assumed known. Even

\footnotetext{
* Their treatment did not assume that the process was Markovian.
} 
after finding $W(t-\tau)$, the problem remained of implementing (4.12), which, being a convolution integral, is not useful for real-time computation of the estimated signal. A dynamic system had to be found whose impulse-response closely matched $W(t-\tau)$.

In engineering circles it gradually became known that almost the only practical way to design WK filters was to assume that both the signal and the noise had "rational spectra" (see, e.g., Phillips, Section 7.4, or Laning and Battin, Section 7.5 ), which is equivalent to assuming that they are conponents of vector GMP's. The Kalman Bucy development starts with this assumption and yields the dynamic system for the filter directly [(4:2) with all coefficient matrices constant]; finding the steady-state solution of (4.3) is thus equivalent to solving a Wiener-Hopf integral equation for rational spectra. Unlike the WK approach, the KB approach handles the multiple-output, multiple-measurement case with ease. However, the wK filter handles measurement noise with rational spectra in a relatively straightforward manner, whereas the KB filter, as originally presented (1961), required that the measurement noise be white (constant spectra). Fortunately, a simple extension of the KB technique enables one to handle "colored" measurement noise even for nonstationary GMP's [cf. Bryson and Johansen (1965)]. In this case the measurement appears directly as part of the estimated state ("feedforward") and, if the measurement noise is sufficiently smooth, the optimum filter may even include differentiators.

Stationary KB filter design may be done using eigenvector decomposition. The Euler-Lagrange equations for the filter are (see Bryson and 110, p. 396): 


$$
\left[\begin{array}{l}
\dot{x} \\
\dot{\lambda}
\end{array}\right]=\left[\begin{array}{ccc}
F & & -\Gamma \mathrm{QT}^{\mathrm{T}} \\
-\mathrm{H}^{\mathrm{T}} \mathrm{R}^{-1} \mathrm{H}, & -\mathrm{F}^{\mathrm{T}}
\end{array}\right]\left[\begin{array}{l}
\mathrm{x} \\
\lambda
\end{array}\right]+\left[\begin{array}{c}
0 \\
\mathrm{H}^{\mathrm{T}} \mathrm{R}^{-1} \mathrm{z}
\end{array}\right] \text {. }
$$

If we let $T_{+}$be the $2 n \times n$ matrix consisting of the $n$ eigenvectors of (4.13) corresponding to the eigenvectors with positive real parts, then

$$
P_{\infty}=-x_{+}\left(\Lambda_{+}\right)^{-1} \text { where } T_{+}=\left[\begin{array}{c}
x_{+} \\
\Lambda_{+}
\end{array}\right] \text {. }
$$

The elgenvalues of the error estimates, $\widetilde{x} \triangleq \hat{x}-x$, are the eigenvalues of (4.13) with negative real parts and the eigenvectors are the columns of $\left[\Lambda_{f}\right]^{-1}[$ see, e.g., Bryson and Hall (1971)].

Again, a more revealing form for (4.13) may be given in terms of the Laplace transform of the measurements, $z(s)$, and the measurement estimates, $H \hat{\mathbf{x}}(s)$ :

$$
\left[R+Z(s) Q Z^{T}(-s)\right] R^{-1}[z(s)-H \hat{x}(s)]=z(s)
$$

where $Z(s)=H[s I-F]^{-1} \Gamma$ is the matrix of transfer functions from the disturbance variables to the measurement variables, 1.e.,

$$
z(s)=Z(s) w(s) \text {. }
$$

The corresponding equation for the filter using measurement feedback gains, $K$, may be written:

$$
\left[I+H(S I-F)^{-1} K\right][z-H \hat{x}(s)]=z(s) \text {. }
$$

It follows that the "spectral factorization" equation is: 


$$
\left[R+Z(s) Q Z^{T}(-s)\right]=\left[I+H(s I-F)^{-1} K\right] R\left[I+H(-s I-F)^{-1} K\right]^{T}
$$

which is a $q \times q$ matrix equation (where $z$ is a q-vector). The problem here is to find the $n \times q$ filter gain matrix $K$. Again, by requiring the eigenvalues with negative real parts of (4.13) to be the same as the eigenvalues of (4.17), the single measurement case is solved $(q=1)$. For $q>1$, we require, in addition, that the eigenvectors of (4.17) be parallel to the eigenvectors of (4.15) that correspond to the eigenvalues of (4.13) with positive real parts [see the discussion following (2.13)].

As an example, we design a steady-state filter for the helicopter in hover (see the last part of Section II). We assume a position measurement and a pitch angle measurement, both with jittery noise having correlation times short compared to decay times of the closed-loop eigenvalues; thus they can be modeled as white noise processes. For an RMS position measurement error of 1 meter with a correlation time of .136 sec., the spectral density is

$$
R_{x}=2(1)^{2}(.136)=.272 \mathrm{~m}^{2} \mathrm{sec}
$$

For an RMS pitch angle measurement error of $0.5 \mathrm{deg}$. with a correlation time of 0.10 sec., the spectral density is

$$
R_{\theta}=2\left(\frac{.5}{57.3}\right)^{2}(0.1)=1.53 \times 10^{-5}(\mathrm{rad})^{2} \mathrm{sec}
$$

On the other hand, the correlation time of wind fluctuations is comparable to, or larger than, the decay times of the closed-loop eigenvalues so we model the wind with a first-order shaping filter as 


$$
\tau_{c} \dot{w}+w=q_{w}
$$

where $q_{w}$ is white noise. We have taken $\tau_{c}=5 \mathrm{sec}$. and RMS wind magnitude as $7 \mathrm{msec}^{-1}$; this implies that the spectral density of $q_{w}$ should be

$$
Q=2(7)^{2}(5)=490 \mathrm{~m}^{2} \sec ^{-1} \text {. }
$$

The wind velocity, $w$, is now an additional (fifth) state variable.

The state-feedback gains on $\theta, q, u$, and $x$ are not changed by adding $w$ as a state variable [in the root locus, Figure 4 , a zero is added that just cancels the additional pole at $\left.s=-\left(1 / \tau_{c}\right)\right]$, but an additional gain is added for w, i.e., $\delta=-c_{\theta} \theta-c_{q} \dot{\theta}-c_{u} \dot{x}-c_{x} x-c_{w} w \ldots$ It is straightforward to show that

$$
C_{w}=B^{-1} G^{T} S_{w}
$$

where

$$
\left[\frac{1}{\tau_{c}} I-\left(F-\mathrm{GB}^{-1} \mathrm{G}^{\mathrm{T}} \mathrm{S}_{\infty}\right)^{\mathrm{T}}\right] \mathrm{S}_{\mathrm{w}}=\mathrm{S}_{\infty} \Gamma \text {. }
$$

For the numbers used here, this gives

$$
C_{w}=-.00195 \mathrm{~m}^{-1} \mathrm{sec} \text {. }
$$

The filter design, on the other hand, is significantly affected by adding the shaping filter. The root square characteristic equation in the form (4.15) is given by

$$
\left|R+Z(s) Q Z^{T}(-s)\right|=0
$$

where

$$
R=\left[\begin{array}{ll}
R_{x} & 0 \\
0 & R_{\theta}
\end{array}\right], \quad \mathrm{Z}(\mathrm{s})=\left[\begin{array}{c}
\mathrm{Z}_{\mathrm{x}}(\mathrm{s}) \cdot \\
\mathrm{z}_{\theta}(\mathrm{s})
\end{array}\right],
$$




$$
\begin{aligned}
& z_{x}(s) \triangleq \frac{x(s)}{q_{w}(s)}=\frac{-\sigma_{2}\left[s^{2}+\left(\sigma_{1}-\frac{a_{1} a_{2}}{\sigma_{2}}\right) s+\frac{a_{1} g^{2}}{\sigma_{2}}\right]}{\left(\tau_{c} s+1\right) \Delta_{0}(s)}, \\
& z_{\theta}(s)=\frac{\theta(s)}{q_{w}(s)}=\frac{-a_{1} s^{2}}{\left(\tau_{c} s+1\right) \Delta_{o}(s)},
\end{aligned}
$$

and $\Delta_{0}(s)$ is given below (2.16). Thus (4.23) may be written as:

$$
\frac{\mathbf{R}_{\theta}}{\mathbf{Q}}+\mathrm{z}_{\theta}(\mathbf{s}) \cdot \mathrm{z}_{\theta}(-\mathbf{s})+\frac{\mathrm{R}_{\theta}}{\mathbf{R}_{x}} \mathrm{z}_{\mathbf{x}}(\mathrm{s}) \mathrm{z}_{\mathbf{x}}(-\mathrm{s})=0
$$

A root locus of $(4.25 a)$ with $Q / R_{\theta}$ as the parameter is shown in Figure 9 for the numerical values of Table 1 ,

$$
\frac{\mathrm{R}_{\theta}}{\mathrm{R}_{\mathrm{x}}}=\frac{1.525 \times 10^{-5}}{.272} \mathrm{~m}^{-2},
$$

and $\tau_{c}=.5 \mathrm{sec}$. The "zeros" of the root locus are at $.190 \pm .193 \mathrm{j} \mathrm{sec}^{-1}$, and the "poles" are the open-1oop poles of Table 1, the "wind pole" at $s=-0.2 \mathrm{sec}^{-1}$, and their reflections in the imaginary axis. For $Q=490$ $\mathrm{m}^{2} \mathrm{sec}^{-1}$; the eigenvalues of the filter estimate errors are given in Table 2 , as well as at the corresponding filter gains and predicted RMS values for the filter estimate errors. Table 3 shows the dynamic compensator, comprised of the filter and the estimated state feedback to rotor tilt, $\delta$.

Even for $Q=0$, a stable filter is designed by this technique. The two unstable complex roots of the open-loop system are "reflected" across the imaginary axis, while the stable real roots and the zero root remain unchanged. The corresponding filter gains and RMS estimate errors are:

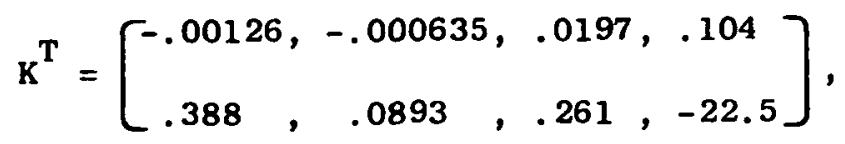

$$
\begin{aligned}
& \tilde{\theta}=.083 \mathrm{deg}, \tilde{q}=.029 \mathrm{deg} \sec ^{-1}, \tilde{u}=.026 \mathrm{msec}^{-1}, \tilde{x}=.072 \mathrm{~m} .
\end{aligned}
$$


It is not always possible to find a satisfactory steady-state filter by these techniques, even when the system is completely observable. If there are any stable (or neutrally stable) modes of the open-loop system that are "uncontrollable" by the disturbances," then the steady-state variance and covariances of these modes will be zero, i.e., the optimum time-varying filter will have estimated them exactly by the time it reaches the statistical steady-state. As a result, the steady-state gains for this mode will be zero; this may produce an unsatisfactory filter, particularly if the undisturbable mode is neutrally stable or only marginally stable (this will show up as a zero or very small eigenvalue for the steady-state filter). A straightforward remedy for this situation is to divide the system into disturbable and undisturbable modes, and then (a) design a KB filter for the disturbable modes neglecting the coupling to the undisturbable modes (i.e., treat them as known forcing functions); then (b) design an "observer" for the undisturbable modes, neglecting the coupling to the disturbable modes, with eigenvalues very much less than the eigenvalues of the filter in (a), but large enough so that errors in these modes will die out rapidly enough to be satisfactory [see, e.g., Bryson and Luenberger (1970) for observer design].

The following example illustrates a neutrally stable, undisturbable mode. An inverted pendulum is to be stabilized by torquing a wheel mounted at the tip of the pendulum as shown in the sketch below. A random wind blows on the pendulum rod but produces no torque on the wheel, so the system model is reasonably well described by

\footnotetext{
"Undisturbable" would be a better word than "uncontrollable" in this context.
} 


$$
\begin{aligned}
& \dot{\theta}=\mathrm{q} \\
& \dot{\mathbf{q}}=\mathrm{n}^{2} \theta+\mathrm{u}+\mathrm{w} \\
& \dot{\mathbf{r}}=-\mathrm{au}
\end{aligned}
$$

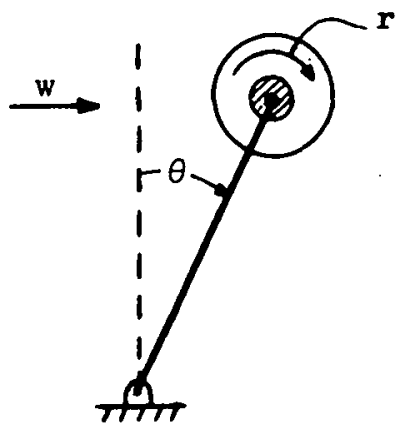

where $\mathbf{r}=$ angular velocity of wheel, $u$ is proportional to the torque applied to the wheel, and $w$ is proportional to the wind velocity. Clearly, the wheel is undisturbable by the wind but $r$ is observable if $r$ or $r-q$ is measured; in principle, then, a nonstationary filter could bring $\hat{\mathbf{r}}-\mathbf{r}$ to zero exactly after a long time even with a very noisy measurement. The steady-state of such a filter would have zero gain on the measurements in the $\dot{\hat{\boldsymbol{r}}}$ channel, i.e., no attention would be pald to new measurement data. v. MEAN SQUARE BEHAVIOR OF SYSTEMS WITH FILTER/STATE-FEEDBACK CONTROLLERS As discussed in the introduction, the control system that minimizes the quadratic criterion (2.3) on the average, in the presence of random disturbances, is constructed by using the state-feedback control gains of (2.5) on the estimated state from the filter (4.2), 1.e.,

$$
\mathbf{u}=-\dot{c} \hat{\mathbf{x}}
$$

This combination of filter and state-feedback gains (see Fig. 1.2) constitutes a "compensator" in the language of classical controller design.

The system, controlled by this compensator, with random forcing functions, $w(t)$, random errors in the sensor signals, $v(t)$, and random initial conditions, $x\left(t_{0}\right)$, is itself a gauss-markov process. Hence, its mean and mean-square behavior can be predicted using (3.9) and (3.10), by considering 
the state vector to be $\left[\begin{array}{c}x \\ -\hat{x} \\ x\end{array}\right]$ and the disturbance vector to be $\left[\begin{array}{c}w \\ v\end{array}\right]$. $\hat{x}$ is thus treated as the "state" of the compensator. However, this prediction is actually simpler than just indicated if we use $\left[\begin{array}{l}\tilde{x} \\ -\hat{\hat{x}}\end{array}\right]$ as the state vector, where $\hat{x}=\hat{x}-x=f i l t e r$ estimate error, because $\hat{x}$ does not depend on $\hat{x}$ :

$$
\begin{aligned}
& \dot{\dot{x}}=(F-K H) \hat{x}+K v-\Gamma w \quad ; \tilde{x}\left(t_{0}\right)=-x\left(t_{0}\right) \\
& \dot{\hat{x}}=(F-G C) \hat{x}-K H \tilde{x}+K v \quad ; \hat{x}\left(t_{0}\right)=0
\end{aligned}
$$

where we have used

$$
\begin{aligned}
& \mathrm{z}=\mathrm{Hx}+\mathrm{v}, \\
& \mathrm{u}=-\mathrm{C} \hat{\mathbf{x}} .
\end{aligned}
$$

Since $E\left[\left[\tilde{x}\left(t_{0}\right)\right]\left[\hat{x}\left(t_{o}\right)\right]^{T}\right\}=0$, it follows from (5.2) that

$$
E\left([\tilde{x}(t)][\hat{x}(t)]^{T}\right\}=0 \text { for } t \geq t_{0} .
$$

Therefore

$$
E\left\{[x(t)]\left[x(t)^{T}\right\} \equiv E\left\{(\hat{x}-x)(\hat{x}-x)^{T}\right\}=E\left(\hat{x} \hat{x}{ }^{T}\right)+E\left(\tilde{x} \tilde{x}^{T}\right),\right.
$$

or

$$
x=\hat{x}+P
$$

where

$$
X=E\left(\mathbf{x} \mathbf{x}^{T}\right), \hat{\mathbf{X}}=E\left(\hat{\mathbf{x}} \hat{\mathbf{x}}^{\mathrm{T}}\right), \quad P=E\left(\mathbf{x} \tilde{\mathbf{x}}^{\mathrm{T}}\right) .
$$

Now $P$ is given by $(4.3)$ and it follows directly from $(3.8),(5.2)$, and (5.4) that

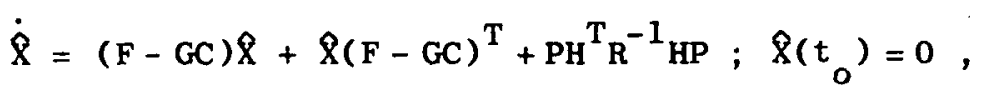

which is a linear matrix equation, since $\mathrm{PH}^{\mathrm{T}} \mathrm{R}^{-1} \mathrm{HP}$ is a known forcing function [1.e., it can be pre-computed from (4.3)]. From (5.3) and (5.5) it follows that

$$
E\left[u(t) u^{T}(t)\right]=c \hat{x} c^{T}
$$

For the statistically stationary case, $\dot{\mathrm{p}}=0, \dot{\hat{x}}=0$, and (5.6) becomes a set of $\frac{1}{2} n(n+1)$ simultaneous linear equations. For even 
moderate values of $n$, solution of that many linear equations is not a trivial task.

For the helicopter example used in sections II and IV, with the data of Tables 1 and 2 , the predicted RMS state and control deviations in the gusty wind (RMS $7 \mathrm{msec}^{-1}$, correlation time 5 sec.) using the state-feedback filter compensator are given in Table 4.

To show the importance of both sensors in the example, we designed a controller using only the posttion sensor (1t is not possible, of course, to use only the pitch angle sensor, since then the position error can not be estimated). For the same position sensor accuracy and the same gusty wind, the predicted RMS deviations were $\theta=24.4$ deg., $x=4.76 \mathrm{~m}, \delta=10.4 \mathrm{deg}$. which would be quite unacceptable. This shows that the pitch angle sensor is essential.

The overall compensator transfer function, from the measurements, $z(s)$, to the controls, $u(s)$, for the stationary case is given by

$$
u(s)=-C[S I-F+G C+K H]^{-1} K z(s)
$$

Some of the poles and zeroes of this transfer function may have positive real parts, i.e., the compensator does not necessarily have "minimum phase". (Most classical compensator designs have minimum phase.) Thus, the compensator (5.8) will, in general, require active logic components, i.e., it cannot be built just from passive components like resistors and capacitors. However, the elgenvalues (poles) of the controlled system are simply the elgenvalues of the state feedback controller, F-GC, plus the eigenvalues of the Filter, F-KH, all of which have negative real parts. *

Thus, (5.8) may obscure the stability of the controlled system. 


\section{SUMMARY}

The current theory available for designing controllers for dynamic systems subjected to random disturbances may be summarized as follows:

(a) Model the system by a set of coupled first-order ordinary differential equations (i.e., put them in state vector form).

(b) Linearize the system about a nominal path or operating condition.

(c) Design a linear state-feedback controller using weighting factors in a quadratic performance index as described in Section II.

(d) Model the random disturbances as Gauss-Markov processes, using shaping filters if necessary.

(e) Design a Kalman-Bucy filter to estimate the state vector from the available measurements as described in section IV.

(f) Cascade the filter and the state-feedback controller, i.e., use state-feedback on the estimated state vector. This is the optimal "compensator" for the system.

(g) Predict the RMS (root mean square) response of the controlled system in the presence of the random disturbances using the methods of section $V$. If the response is unsatisfactory, modify the compensator by changing weighting factors in (c) and/or use additional or more accurate measurements.

\section{ACKNOWLEDGEMENTS}

The author wishes to thank his colleagues, J. V. Breakwe11, D. B. DeBra, J. D. Powell, W. E. Hall, and his students for many valuable suggestions and criticisms of this paper. 


\section{REFERENCES}

B-1 Bryson, A. E., and Ho, Y. C., Applied Optimal Control, XeroxBlaisdell, Lexington, Mass., 1969.

B-2 Bryson, A. E., and Hall, W. E., "Optimal Control and Filter Synthesis by Eigenvector Decomposition," stanford University, SUDAAR Report No. 446, November 1971.

B-3 Bryson, A. E., and Johansen, D. E., "Linear Filtering for TimeVarying Systems Using Measurements Containing Colored Noise," IEEE Trans. Auto. Control, Vol. 10, 4-10 (1965).

B-4 Bryson, A. E., and Luenberger, D. G., "The Synthesis of Regulator Logic Using State-Variable Concepts," Proc. IEEE, Vol. 58, 1803$1811(1970)$.

C-1 Chandrasekar, S., "Stochastic Problems in Physics and Astronomy," Reviews of Modern Physics, Vol. 15, 1-89 (1943).

C-2 Chang, S. S. L., Synthesis of Optimum Control Systems, McGraw-Hill, New York, 1961 .

E-1 Einstein, A., Ann. d. Physik, Vol. 17, 549 (1905) and Vol. 19, 371 (1906).

E-2 Evans, W. R. "Control System Synthesis by Root Locus Method," AIEE Trans. Part II, Vol. 69, 66-69 (1950).

F-1 Francis, J. G. F., "The QR Transformation," Computer Jour., Vo1. 4, 265-271 (1961); Vol. 4, 332-345 (1962).

G-1 Gunckel, T. L., and Franklin, G. F., "A General Solution for Linear Sampled-Data Control Systems," Trans. ASME, Jour. Basic Engr., Vol. 85-D, 197-201 (1963). 
H-1 Hall, W. E., "Computational Methods for the Synthesis of RotaryWing VTOL Aircraft Control Systems," Ph.D. Dissertation, Stanford Univ., Stanford, Calif., 1971.

J-1 Joseph, P. D., and Tou, J. T., "On Linear Control Theory," Trans. A IEE (Applications and Industry), Vo1. 80, 193-196 (1961).

K-1 Kalman, R. E., and Bertram, J. E., "General Synthesis Procedure for Computer Control of Single and Multiloop Linear Systems," Trans. AIEE, Vol. 77 (1958).

K-2 Kalman, R. E., and Bucy, R., "New Results in Linear Filtering and Prediction," Trans. ASME, Vol. 83D, 95-108 (1961).

K-3 Kalman, R. E., and Englar, T. S., "A User's Manual for the AutoMatic Synthesis Program," NASA CR-475, June 1966.

K-4 Kalman, R. E., Ho, Y. C., and Narendra, K. S. "Controllability of Linear Dynamic Systems," Contrib. to Differential Eqns., Vol. $1,189-213$ (1963).

K-5 Khintchine, A. Ya., "Correlation Theory of Stationary Stochastic Processes," Math. Annalen, Vol. 109, 604-615 (1934). Also Progress of Math. Sci., USSR, No. 5, 42-51 (1938).

K-6 Kolmogorov, A. N., Foundations of the Theory of Probability, Chelsea, New York (1931).

K-7 Kolmogorov, A. N., "Interpolation and Extrapolation of Stationary Random Sequences," Bul1. Moscow Univ., USSR, Ser. Math. Vo1. 5, 13-14 (1941).

L-1 Langevin, P., Comptes Rendus, Vol. 146, 530 (1908).

L-2 Laning, J. H., and Battin, R. H., Random Processes in Automatic Control, McGraw-Hill, New York, 1958. 
L-3 Letov, A. M., "Analytical Controller Design" (3 parts), Automation and Remote Control, Vol. 21, 1960.

M-1 Markov, A. A., Wahrscheinlichkeitsrechnung, Le1pzig, 1912.

M-2 McFarlane, A. G. J., "An Eigenvector Solution of the Linear Optimal Regulator Problem," Jour. Electronics and Control, Vol. 14, 643654 (1963).

N-1 Newton, G. C., Gould, L. A., and Kaiser, J. F., Analytical Design of Linear Feedback Control, Wiley, New York, 1957.

P-1 Planck, M., Sitz. der Preuss., Akad., p. 324, 1917.

P-2 Phillips, R. S., Chapters 6 and 7 of Theory of Servomechanisms, ed. by James, Nichols, and Phillips; McGraw-H111, New York (1947).

P-3 Potter, J. E., "A Guidance-Navigation Separation Theorem," MIT Expt. Astronomy Lab. Rep. RE-11, Aug. 1964.

P-4 Potter, J. E., "Matrix Quadratic Solutions," SIAM Jour. Appl. Math., Vo1. 14, 496-501 (1966).

R-1 Rice, S. O., "Mathematical Analysis of Random No1se," Bell system Technical Journal, Vols 23 and 24, 1-162 (1944-45).

R-2. Rynaski, E. G. and Whitbeck, R. F., "Theory and Application of Linear Opt1mal Control," Cornell Aero. Lab. Report IH-1943-F-1, 193 pp., Oct. 1965. (Also AFFDL-TR-65-28).

U-1. Uhlenbeck, G. E. and Ornstein, L. S., "On the Theory of Brownian Motion," Physical Review, Vol. 36, 823-841 (1930).

W-1 Wang, M. C., and Uhlenbeck, G. E., "On the Theory of Brownian Motion II," Reviews of Modern Physics, Vol. 17, 323-342 (1945).

W-2 Wiener, N., "Generalized Harmonic Analysis," Acta Mathemtatica, Vol. 55, 117-258 (1930). 
W-3 Wiener, N., "The Extrapolation, Interpolation, and Smoothing of Stationary Time Series with Engineering Applications," MIT Radiation Lab, Rpt., Feb, 1942 (published as a book, 1949, by Wiley, New York).

W-4 Wold, H., A Study in the Analysis of Stationary Time Series, Almquist and Wiskell, Uppsala, 1938.

\section{BIBLIOGRAPHY}

A-1 Aström, K. J., Introduction to stochastic Control Theory,

B-1 Boguslavski, I. A., Methods of Navigation and Control with Incomplete Statistical Information, Moscow, 1970 (In Russian). Academic Press, New York, 1970.

L-1 Leondes, C. T. (Ed.), Theory and Applications of Kalman Filtering, AGA Rograph No. 139, National Technical Information Service, Springfield, Virginia 22151, Feb. 1970.

L-2 Letov, A. M., Dynamics and Control of Flight, Moscow, 1969 (In Russian).

M-1 Meditch, J. S., Stochastic Optimal Linear Estimation and Control, McGraw-Hill, New York, 1969.

W-1 Wilkerson, J. H., Martin, R. S., Peters, G., "The QR Algorithm for Real Hessenberg Matrices," Numer. Math., Vol. 14, 219-231 (1970). 


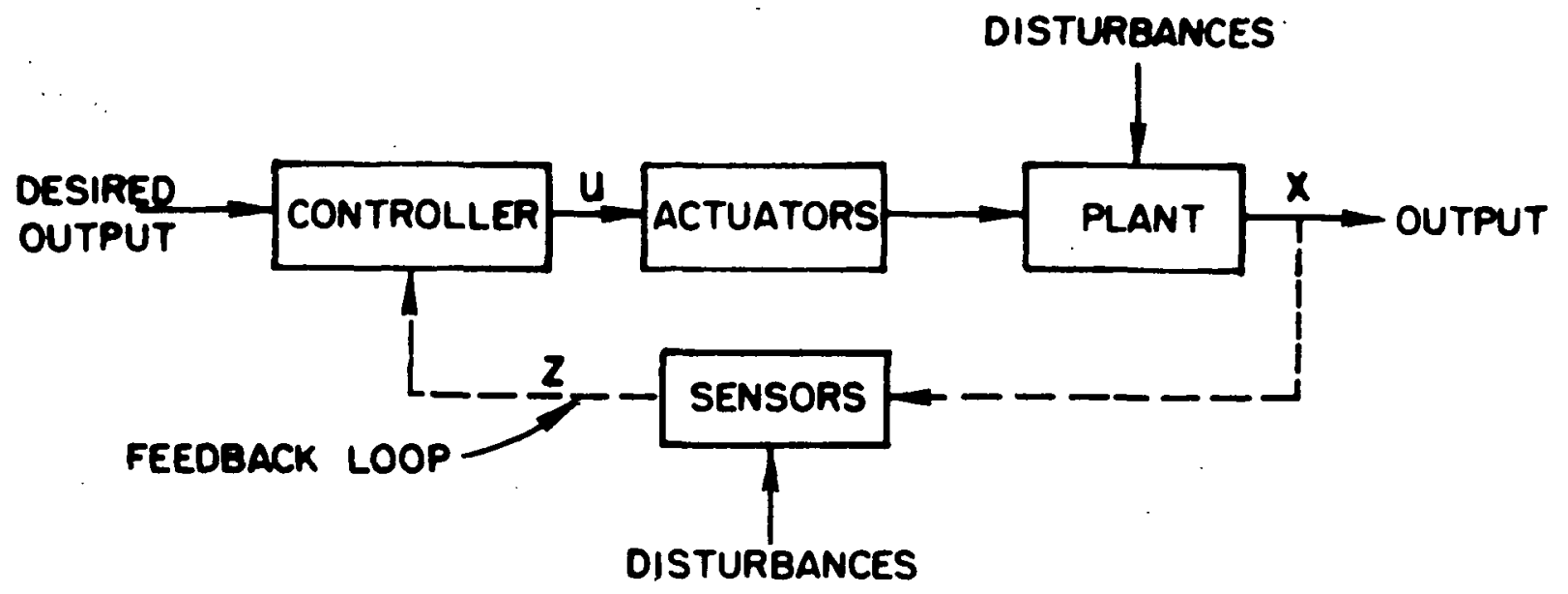

FIG. 1 .

Block Diagram; Open-Loop (Programmed) Control Has No Feedback Loop Closed-Loop Control Has Feedback Loop

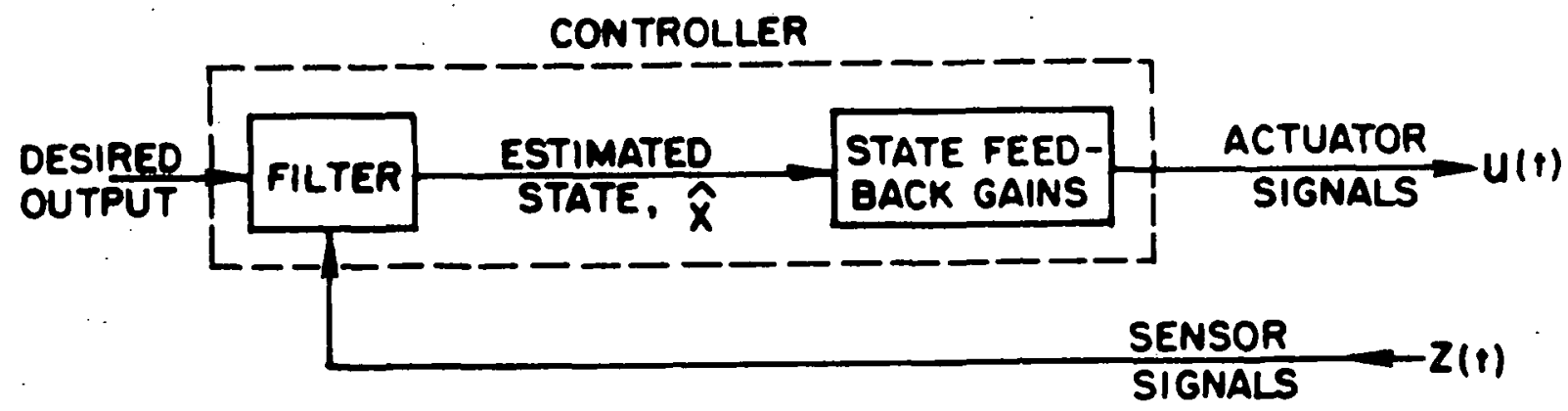

FIG. 2

Block Diagram; Dynamic Compensator Formed from Filter and State-Feedback Gains 


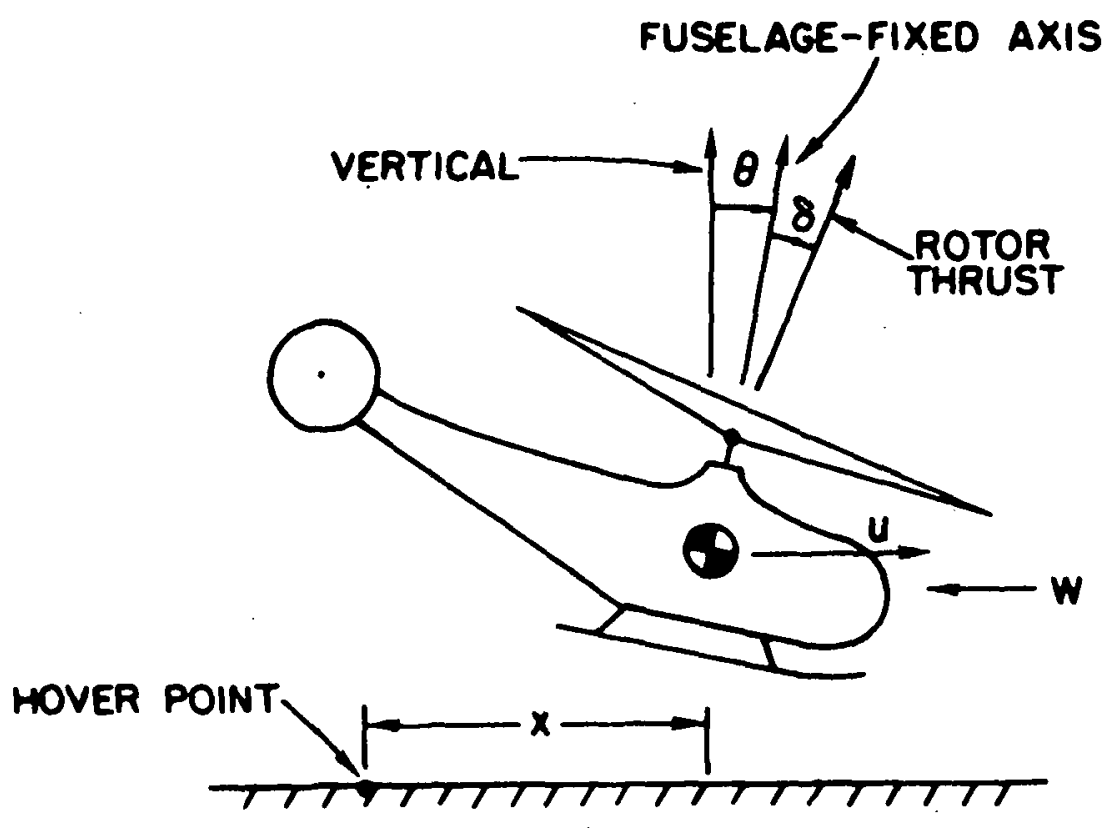

FIG. 3 .

Nomenclature and Reference Axes

for Helicopter Hover Example 


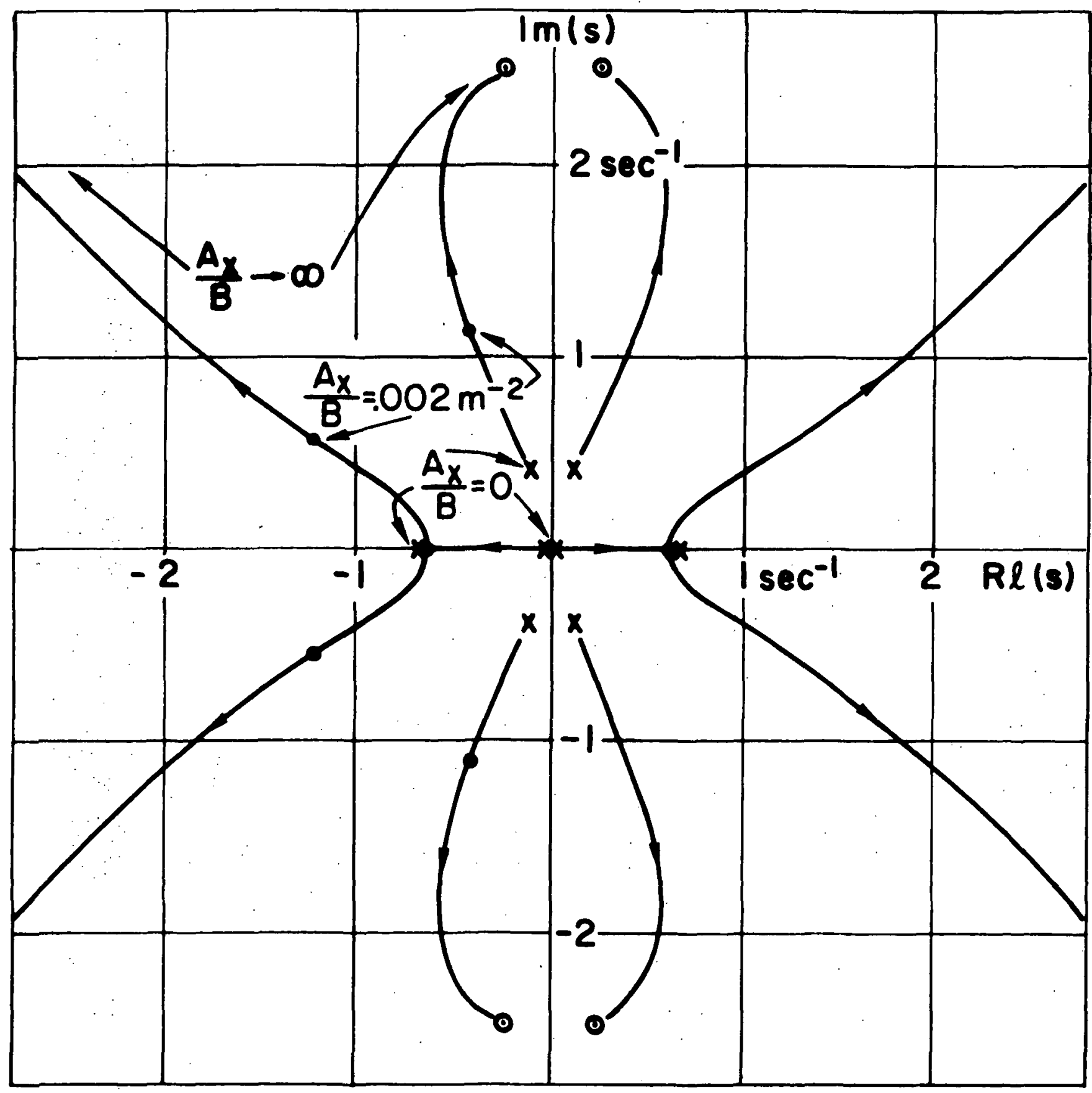

FIG, 4 .

Root Locus for Position Regulator, Helicopter in Hover, with $A_{x} / B$ as Parameter 


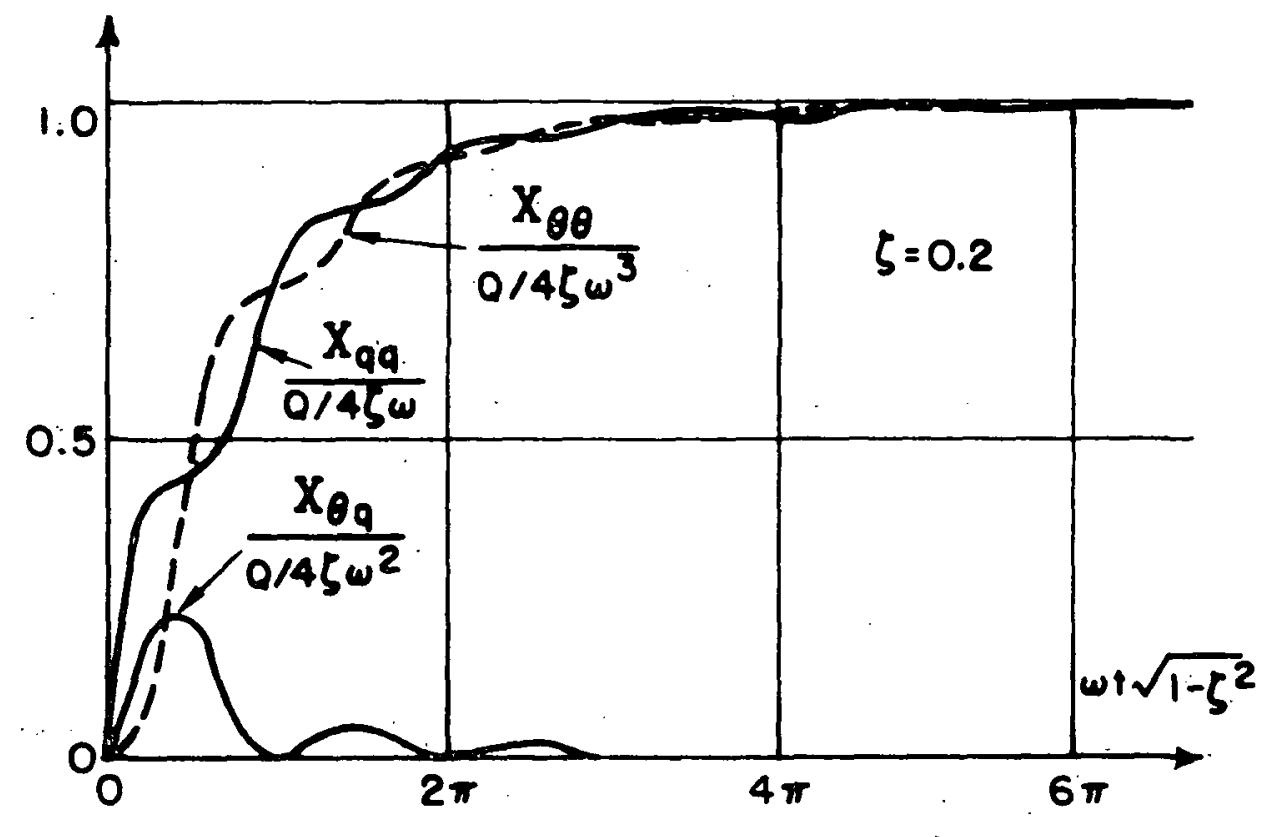

FIG. 5 .

Pendulum in Random Wind of Spectral Density $Q$;

Variance and Covariance Histories, Starting with Zero Variance

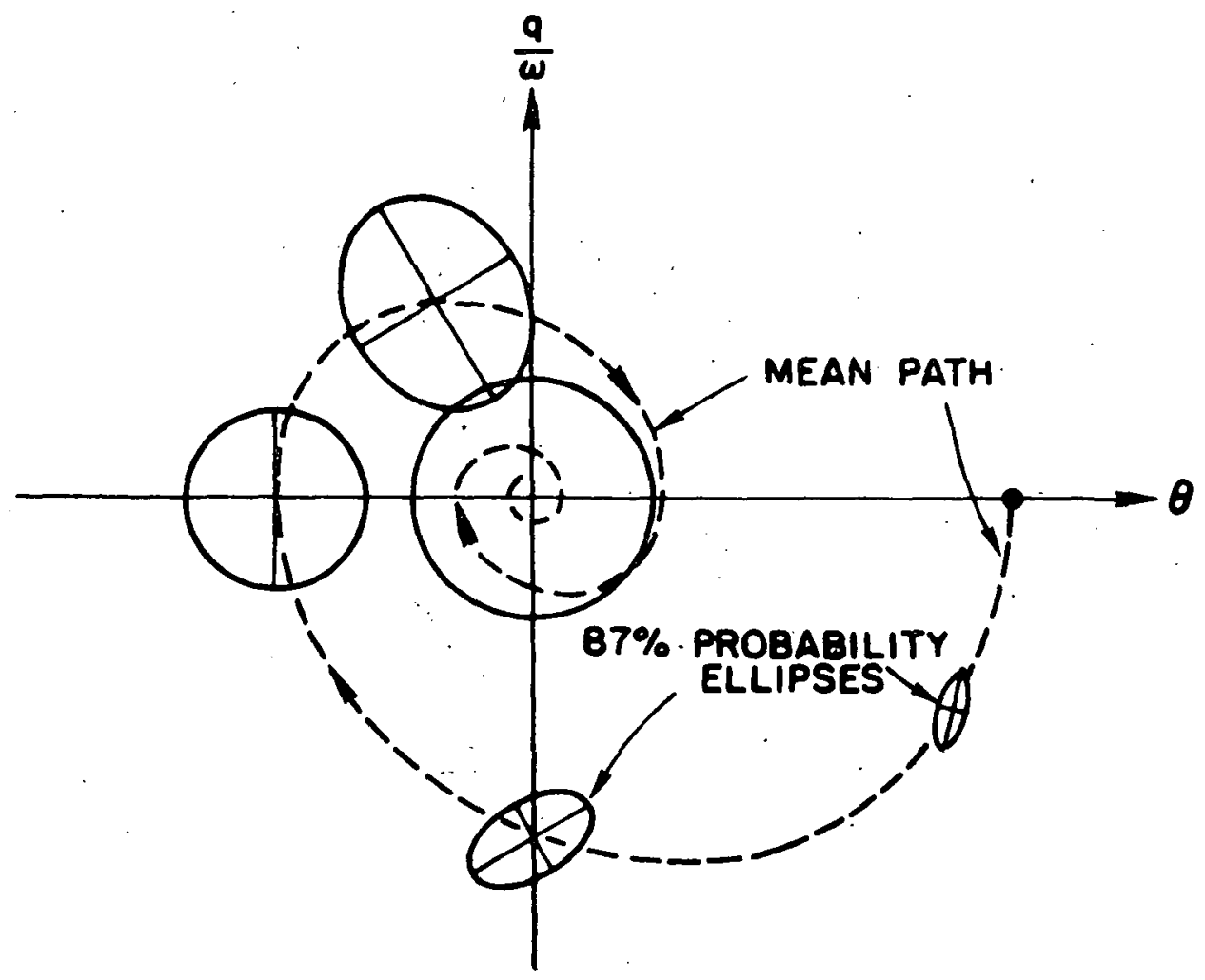

FIG. 6.

Pendulum in Random Wind;

Mean Angular Velocity (q) vs. Angular Deflection $(\theta)$, with $87 \%$ Probability Ellipses at Selected Points on Path 


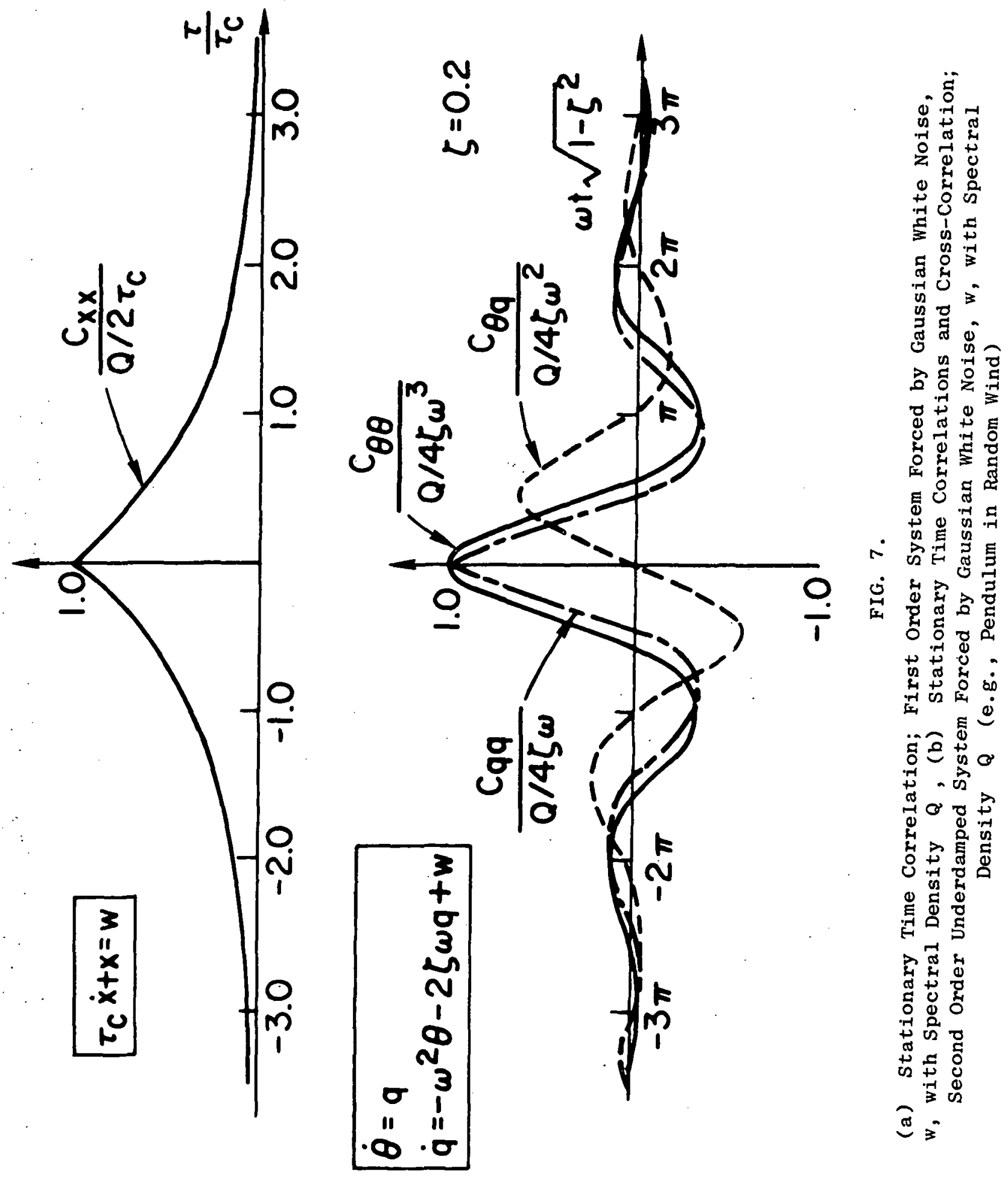




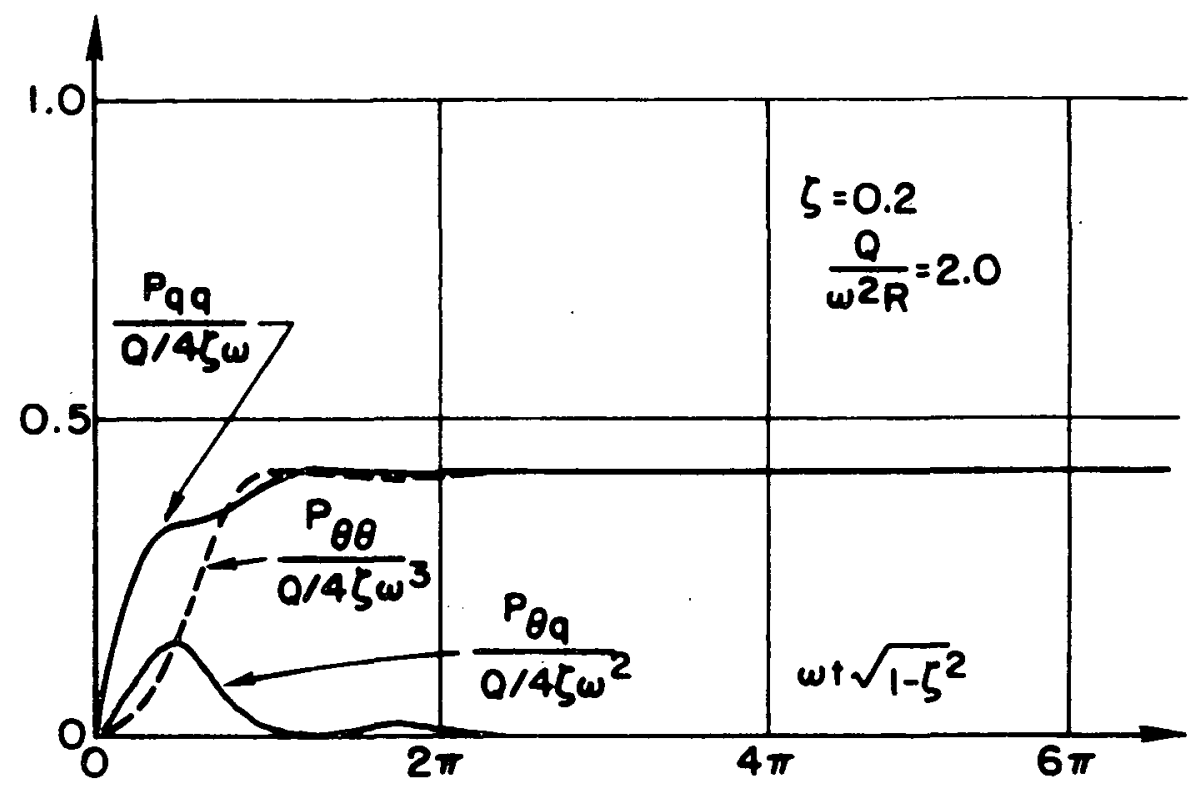

FIG. 8.

Pendulum in Random Wing - Estimate Error Variance and Covariance Histories Using Measurement of $q$ with Additive White Noise Having Spectral Density $R$ 


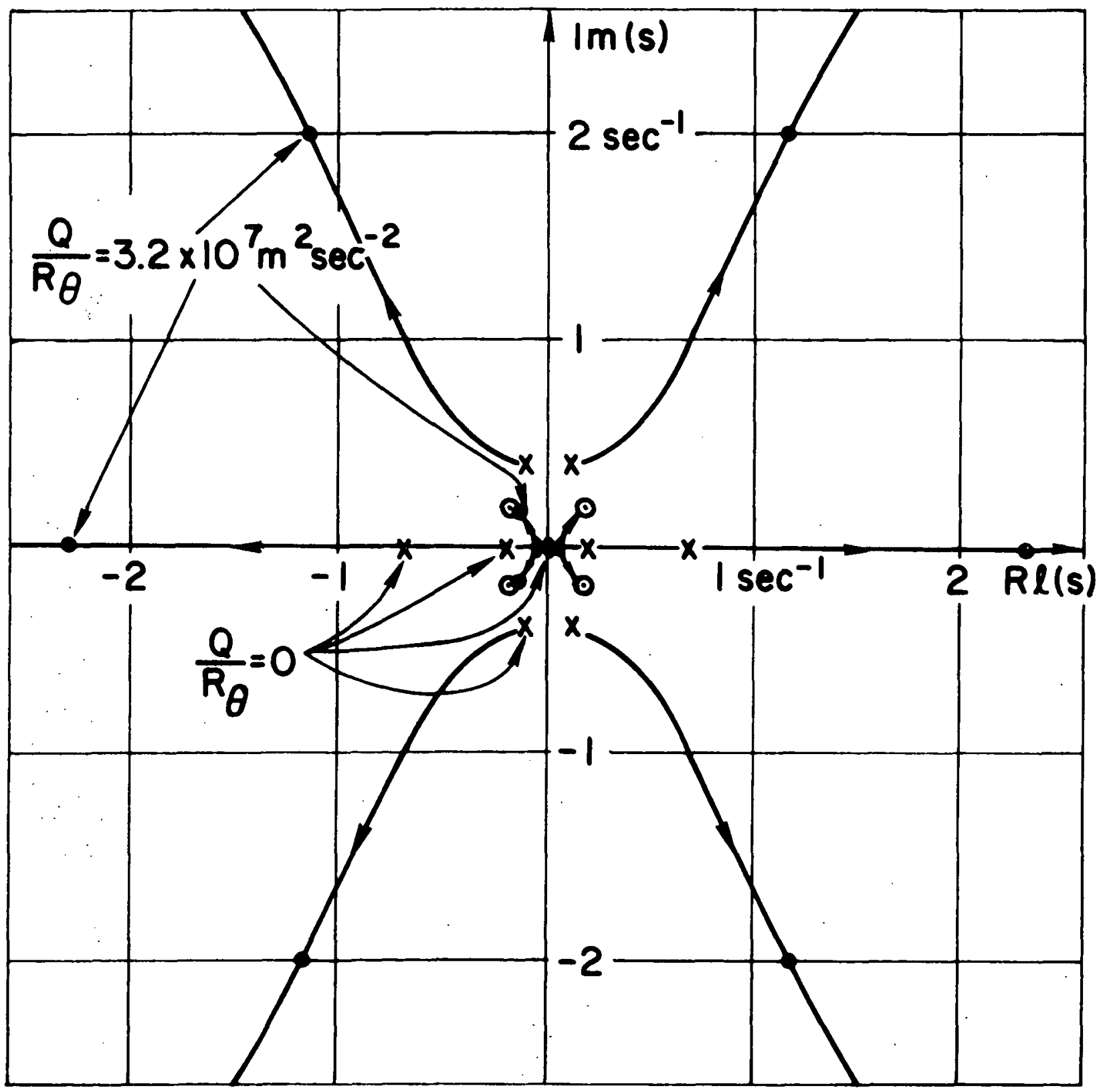

FIG. 9 .

Root Locus for $(x, \theta)$ Measurement Filter, Helicopter in Hover, with $Q / R \theta$ as Parameter, $\left(R_{x} / \theta\right)=5.6 \times 10^{-5} \mathrm{~m}^{-2}$ 
REGULATOR DESIGN FOR A TYPICAL TRANSPORT HELICOPTER

$$
\begin{aligned}
& \text { Coefficients: } \sigma_{1}=.415 \mathrm{sec}^{-1} \quad, \sigma_{2}=.0198 \mathrm{sec}^{-1}, \mathrm{n}=6.27 \mathrm{sec}^{-2} \text {, } \\
& a_{1}=.0111 \mathrm{~m}^{-1} \mathrm{sec}^{-1}, a_{2}=1.43 \mathrm{msec}^{-1}, \mathrm{~g}=9.80 \mathrm{msec}^{-2}
\end{aligned}
$$

Open-Loop Eigenvalues and Eigenvectors*

\begin{tabular}{|l|c|c|c|}
\hline $\mathrm{s}, \mathrm{sec}^{-1}$ & 0 & -.68 & $+.12 \pm .38 \mathrm{j}$ \\
\hline \hline $0, \mathrm{deg}$. & 0 & 2.4 & $.79 \pm .56 \mathrm{j}$ \\
\hline $\mathrm{x}$, meters & 1.0 & 1.0 & 1.0 \\
\hline
\end{tabular}

Closed-Loop Eigenvalues and Eigenvectors* for $\left(A_{x} / B\right)=.002 m^{-2}$

\begin{tabular}{|l|c|c|}
\hline $\mathrm{s}, \mathrm{sec}^{-1}$ & $-1.24 \pm .56 \mathrm{j}$ & $-.42 \pm 1.14 \mathrm{j}$ \\
\hline \hline$\theta, \mathrm{deg}$. & $8.0 \mp 4.9 \mathrm{j}$ & $-5.4 \mp 8.1 \mathrm{j}$ \\
\hline $\mathrm{x}$, meters & 1.0 & 1.0 \\
\hline
\end{tabular}

Feedback Gains for $\left(A_{x} / B\right)=.002 \mathrm{~m}^{-2}$

\begin{tabular}{|l|l|l|l|}
\hline $\mathrm{c}_{\theta}=.864$ & $\mathrm{c}_{\mathrm{q}}=.325 \mathrm{sec}$. & $\mathrm{c}_{\mathrm{u}}=.0869 \mathrm{~m}^{-1} \mathrm{sec}$. & $\mathrm{c}_{\mathrm{x}}=.0447 \mathrm{~m}^{-1}$ \\
\hline
\end{tabular}

where $\delta=-c_{\theta} \theta-c_{q} q-c_{u} u-c_{x} x$

* The $q$ and $u$ components of the eigenvectors are given by $q=s \theta$, $\mathbf{u}=\mathbf{s x}$. 


\section{TABLE 2}

FILTER DESIGN FOR A TYPICAL TRANSPORT HELICOPTER

[RMS wind $7 \mathrm{msec}^{-1}$ with correl. time $5 \mathrm{sec}, R_{\mathrm{X}}=.272 \mathrm{~m}^{2} \mathrm{sec}, \mathrm{R}_{\theta}=1.52 \times 10^{-5} \mathrm{sec}$ ]

Eigenvalues of the Filter Estimate Errors ( $\left.\mathrm{sec}^{-1}\right)$

\begin{tabular}{|l|l|l|}
\hline-2.34 & $-0.19 \pm 0.19 \mathrm{j}$ & $-1.17 \pm 2.00 \mathrm{j}$ \\
\hline
\end{tabular}

Filter Gains (Units in meters, sec, radians)

\begin{tabular}{|c|c|c|c|c|c|}
\hline $\mathrm{n}$ & $\theta$ & $\mathrm{q}$ & $\mathrm{u}$ & $\mathrm{x}$ & $\mathrm{w}$ \\
\hline $\mathrm{K}_{\mathrm{nx}}$ & .000327 & .000251 & .0735 & .381 & -.128 \\
\hline $\mathrm{K}_{\mathrm{n} 0}$ & 4.05 & 8.21 & 21.4 & 5.94 & -954 \\
\hline
\end{tabular}

RMS Estimate Errors

\begin{tabular}{|l|l|l|l|l|}
\hline$\widetilde{\theta}=0.45 \mathrm{deg}$ & $\tilde{\mathbf{q}}=1.15 \mathrm{deg} \sec ^{-1}$ & $\tilde{\mathbf{u}}=.092 \mathrm{msec}^{-1}$ & $\tilde{\mathbf{x}}=0.32 \mathrm{~m}$ & $\tilde{\mathbf{w}}=3.78 \mathrm{msec}^{-1}$ \\
\hline
\end{tabular} 
DYNAMIC COMPENSATOR FOR A TYPICAL TRANSPORT HELICOPTER

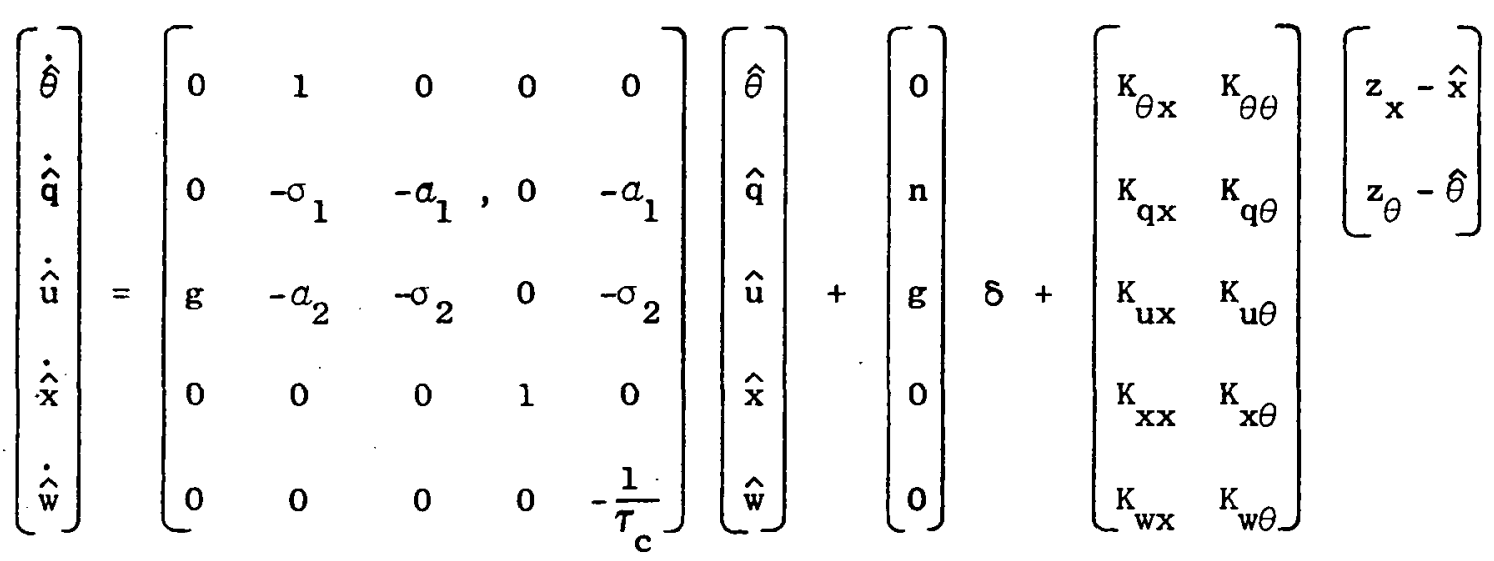

$\delta=-c_{\theta} \hat{\theta}-c_{q} \hat{q}-c_{u} \hat{u}-c_{x} \hat{x}-c_{w} \hat{w}=$ commanded rotor tilt,

$z_{x}=$ measurement of $x$,

$z_{\theta}=$ measurement of $\theta$, and

$C_{n}, K_{n x}, K_{n \theta}$ given in Tables 1 and $2 ; \tau_{c}=5 \mathrm{sec}, C_{w}=-.00195 \mathrm{~m}^{-1} \mathrm{sec}$.

\section{TABLE 4}

PREDICTED RMS STATE AND CONTROL DEVIATIONS OF TYPICAL TRANSPORT HELICOPTER IN A GUSTY WIND USING CONTROLLER OF TABLES I AND 2

\begin{tabular}{|l|l|l|l|}
\hline$\theta=1.95 \mathrm{deg}$ & $\mathrm{q}=2.92 \mathrm{deg} \sec ^{-1}$ & $\mathrm{u}=0.25 \mathrm{msec}^{-1}$ & $\mathrm{x}=0.51 \mathrm{~m}$ \\
\hline
\end{tabular}

$$
\delta=1.33 \mathrm{deg}
$$

